Multitarget Tracking with Doppler Ambiguity 


\title{
MULTITARGET TRACKING WITH DOPPLER AMBIGUITY
}

\author{
BY \\ LI, KAIBING, B.Sc.
}

A THESIS

SUBMITTED TO THE DEPARTMENT OF ELECTRICAL \& COMPUTER ENGINEERING AND THE SCHOOL OF GRADUATE STUDIES OF MCMASTER UNIVERSITY

IN PARTIAL FULFILMENT OF THE REQUIREMENTS FOR THE DEGREE OF Master of Applied SCIEnce

(C) Copyright by Li, Kaibing, September 2010 All Rights Reserved 
Master of Applied Science (2010)

(Electrical \& Computer Engineering)
McMaster University

Hamilton, Ontario, Canada

TITLE: Multitarget Tracking with Doppler Ambiguity

AUTHOR:

Li, Kaibing

B.Sc., (Electrical Engineering)

Huazhong University of Science and Technology, China

SUPERVISOR: Dr. Kirubarajan

NUMBER OF PAGES: ix, 62 
To my mother, who has been supporting me all the time 


\section{Abstract}

In many radar tracking systems with a certain pulse-repetition frequency (PRF), Doppler (or range rate) measurements are available in addition to position measurements. The extra range rate information Doppler measurements provide is able to improve the tracking performance. However, a fundamental problem associated with Pulse-Doppler radars is, especially at low PRFs, range rate ambiguity. This is because Doppler shifts in the frequency spectrum are aliased by a difference of an integer times of PRF. In this case, the observed Doppler measurement shifts from the true range rate by an unknown difference.

In previous works, algorithms to eliminate the Doppler ambiguity based on the Chinese Remainder Theorem have been proposed for radars with multiple PRFs. Time-Frequency Analysis (TFA) using Fast Fourier Transform (FFT) can reduce the ambiguity in the frequency domain.

In this paper, a new approach for multitarget detection and tracking with Doppler ambiguity is presented. Ambiguous Doppler measurements in addition to the position measurements are directly used in data association and tracking. To solve the Doppler ambiguity for single target tracking, three methods are proposed based on UKF, MHT and PDA, respectively. In addition, modifications to Multiple Hypothesis Tracking (MHT) and Joint Integrated Probabilistic Data Association (JIPDA) algorithms to 
resolve Doppler ambiguity in multitarget tracking are considered. Simulation results are preformed to demonstrate the new algorithms with tracking results analysis. 


\section{Acknowledgements}

I would like to thank all my friends, family members and others who encouraged and supported me during the research leading to this thesis.

I would like to express my sincere gratitude to my supervisor, Dr. T.Kirubarajan, for giving me an opportunity to work on a very interesting topic and for providing continuous guidance, advice and support throughout the course of this research work. I also thank Dr. R. Tharmarasa and Dr. Michel Pelletier for their valuable comments and suggestions.

I thank my fellow graduate students in the ECE department, especially all my friends in the ETF Lab, for their help and support. Further, my sincere thanks are due to the ECE department staff, especially Cheryl Gies.

Finally, I would like to thank my family for supporting and encouraging to pursue this degree. 


\section{Contents}

Abstract $\quad$ iv

Acknowledgements $\quad$ vi

1 INTRODUCTION 1

2 OVERVIEW OF TARGET TRACKING 7

2.1 Filtering Algorithms . . . . . . . . . . . . . . . . 7

2.2 Kalman Filter . . . . . . . . . . . . . . . . . . . . . . 9

2.3 Extended Kalman Filter . . . . . . . . . . . . . . . . . . . . . . 10

2.4 Unscented Kalman Filter . . . . . . . . . . . . . . . . . . . . . . . . 11

3 PROBLEM STATEMENT $\quad 15$

3.1 Target State Propagation . . . . . . . . . . . . . . . . . 15

3.2 Target Generated Measurements . . . . . . . . . . . . . . . . . 17

4 SINGLE TARGET TRACKING WITH DOPPLER AMBIGUITY 19

4.1 UKF with Doppler Ambiguity . . . . . . . . . . . . . . . . . 19

4.2 MHT with Doppler ambiguity . . . . . . . . . . . . . . 23

4.3 PDA with Doppler Ambiguity . . . . . . . . . . . . . . . . . 26 
4.3.1 The standard PDA Update . . . . . . . . . . . . . . . 28

4.3.2 Probability of Ambiguity Events. . . . . . . . . . . . 28

\section{MULTITARGET TRACKING WITH DOPPLER AMBIGUITY 30}

5.1 Track-Measurement-Doppler Ambiguity Association . . . . . . . . . . 30

5.2 MHT Algorithm . . . . . . . . . . . . . . . . . . . 33

5.3 JIPDA Algorithm . . . . . . . . . . . . . . . . . . . . . 37

6 SIMULATION AND PERFORMANCE EVALUATION 44

6.1 Single Target . . . . . . . . . . . . . . . . . . . . 44

6.1.1 Simulation Scenario . . . . . . . . . . . . . . . . 44

6.1.2 Performance Evaluation . . . . . . . . . . . . . 45

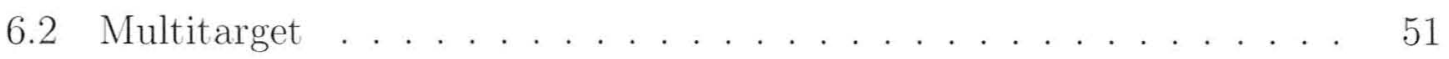

6.2.1 Simulation Scenario . . . . . . . . . . . . . 51

6.2.2 Performance Evaluation . . . . . . . . . . . . 53

$\begin{array}{lll}7 & \text { CONCLUSION } & 57\end{array}$ 


\section{List of Figures}

4.1 Examples of Sigma Points Distribution . . . . . . . . . . . . . . 20

4.2 Example of Evolving and Managing Hypotheses . . . . . . . . . . . . 26

5.1 Example of One Assignment . . . . . . . . . . . . . . . . . . . 31

5.2 Assignment Problem . . . . . . . . . . . . . . . . . . . 32

5.3 Example of Association with Doppler Measurement . . . . . . . . . 35

6.1 UKF tracking results . . . . . . . . . . . . . . . . . . . . . . 46

6.2 RMSE for UKF and MHT . . . . . . . . . . . . . . . 50

6.3 Target Trajectory and Measurements . . . . . . . . . . . . . . 52

6.4 Tracking Results . . . . . . . . . . . . . . . . . . . . 53 


\section{Chapter 1}

\section{INTRODUCTION}

In many radar or sonar tracking systems, the state of interest typically includes target position and velocity components. In addition to position measurements, target Doppler or range rate measurement may provide further information about a targets second order kinematic state. where the Doppler effect is used to determine the relative velocity of objects or whether the target is stationary or moving (Koch, 2002). Pulses of RF energy returning from the target are processed to measure the frequency shift between carrier cycles in each pulse and the original transmitted frequency. Therefore, the Doppler measurement can be incorporated into the tracker to enhance tracking performance. On the other hand, in a coherent pulsed Doppler radar, ambiguity can occur in both range and radial velocity measurements (Alabaster et al., 2003) (Trunk and Brockett, 1993). The ambiguity domain remains constant for a fixed carrier frequency. As the pulse repetition frequency (PRF) increases, the ambiguity moves from radial velocity to range measurements. In general, a compromise is made between high PRF and low PRF, both of which can cause side effects in range and range rate, respectively (Zhou et al., 2006). The medium PRF 
radar system allow all-round measurements of both range and Doppler of targets in high clutter environment to be made (Hughes and Lewis, 2009). Such radars use waveforms that are ambiguous in range, Doppler or both. In most case, the main purpose of the surveillance radars is the observation of the targets in the area of interest. Therefore, low PRF is chosen to make an accurate range measurement, but it leads to the ambiguity in the range rate. In this paper, only the Doppler ambiguity is considered.

In previous works, several methods have been proposed to introduce the Doppler measurement into the tracking step which aims to improve the estimation performance. In (Smith, 2008), the said improvement, for both the position and velocity estimation, was illustrated by analysis of the fundamental lower bound of the estimation error covariance (Zhang et al., 2005) (Tharmarasa et al., 2007), which indicated that the lower bound decreases proportional to the standard deviation of the error in the Doppler component. In Niu et al. (2002), the range rate information is extracted from the radar wave form in a linearly frequency-modulated (LFM) pulse radar system, and the corroboration in the new range rate measurements offers an good tracking performance. In (Yeom et al., 2004) (Wang et al., 2009) (Wang et al., 2007), instead of the traditional "two-point" track initialization, the "one poin" track initialization has been presented, where the Doppler measurements are incorporated here to reduce the velocity uncertainty for the initiated track states. The proposed track initiation method is capable of reducing the computational requirements of the system compared with the classical one. In (Tobias and Lanterman, 2005), the PHD particle filter handles ghost targets and it has improved tracking performance when incorporating Doppler measurements along with range measurements. Meanwhile, 
the negative correlation between the measurement error of range and that of Doppler has been proved in the case of commonly used upsweep chirp or linear FM waveform (Bar-Shalom, 2000). In (Wang et al., 2009) (Wang et al., 2007), the use of the Doppler measurement for PDA in multitarget tracking has been addressed without the use of nonlinear filter. The position and Doppler measurements are processed with independent linear filters, both of which contribute to the data association of PDA. However, the complexity of the target maneuvers and the geometry of the radar environment (Blackman, 1986), either polar or spherical system, indicate that the tracking system design needs to employ nonlinear filtering techniques. Using measurements of Doppler information directly in target state estimation also leads to a nonlinear filter. The simplest and oldest nonlinear filtering technique, Extended Kalman Filter (EKF), has been commonly used in the previous works (Jazwinski, 1970) (Bar-Shalom et al., 2001). However, the EKF suffers a number of serious limitations (Julier and Uhlmann, 2004), such as, linearized transformations are only reliable if the error propagation can be well approximated linearly; the calculating Jacobian matrices could be a very difficult and error-prone process; or even worse, the Jacobian matrix does not exist (Tugnait, 1981), which holds for the model of the ambiguous Doppler measurement. Therefore EKF often results in major errors in the estimated statistics of the posterior distributions of the states. Unlike the EKF, the unscented Kalman filter (UKF), which is first applied in (Julier and Uhlmann, 2004) (Wan and Van Der Merwe, 2000), uses the true nonlinear model for state propagation or observation rather than a linearly approximation. When propagated by the true non-linear model, the posterior mean and covariance can be accurately estimated, while errors only exist in the third or higher order. 
On the other hand, above-mentioned works only incorporate the Doppler measurement without any ambiguity into tracking. The fundamental problem associated with Pulse-Doppler radars, especially at low PRFs, is range rate ambiguity. This is because Doppler shifts in the frequency spectrum will be aliased by a difference of an integer times of PRF. In this case, the observed Doppler measurement differs by $n_{d} \times v_{f}$ from the true range rate of the target, where $n_{d}$ is the unknown ambiguity order and $v_{f}$ is the first blind velocity, which depends on the PRF. To solve this ambiguity problem, multiple PRFs are often used (Hughes and Lewis, 2009) (Hovanessian, 1976), so that the ambiguity could be solved by the Chinese Remainder Theorem with the appropriate selection of PRF. However, the task of solving ambiguity would be time and resource consuming. On the other hand, various works have presented to solve this problem in frequency domain (Xia, 1999) (Ferrari et al., 1997). The folded frequency of the target signal can be estimated by averaging the folded frequency estimates for each PRF. And the ambiguity order is the integer part of the Doppler frequency, which is estimated by using the quasi-maximum likelihood criterion, with a fast implementation based on the Fast Fourier Transform (FFT). Methods in (Koch, 2002) (Zhou et al., 2006) solve the Doppler ambiguity by analyzing the waveform of the receiving radar signal. However, all the proposed methods to solve the ambiguity rely on the choice of the particular values of the PRFs, multiple measurements for a singe target have to be processed. And all the above mentioned methods are implemented independently from the tracking or data association step, which yields to extra computation load to the tracking system.

In this paper, based on UKF, multiple hypothesis tracking (MHT) and probabilistic data association (PDA), three methods of solving ambiguity in the tracking level 
have been developed, theoretically independent of the choice of particular PRF. The UKF is modified to handle the ambiguous Doppler measurement. Although with limitations of maneuverability and measurement accuracy, the modified UKF can achieve better tracking performance in general. On the other hand, MHT and PDA, both of which used to solve the measurement-to-track association problem, are modified here to achieve optimal or suboptimal solution for the Doppler ambiguity problem. The MHT algorithm is brought out (Reid, 1979) to deal the measurement-to-track association in tracking multiple targets. The Key idea of MHT is exhaustively searching all possible association events from time 1 to time $\mathrm{k}$ and find the one with greatest probability. Instead of measurement-to-track association ambiguity, the MHT algorithm is applied to solve the Doppler ambiguity here. For the multitarget tracking, based on the original MHT, sub-hypotheses are generated according to the Doppler ambiguity for each pair of track-measurement assignment. The exponential increasing number of hypotheses would results in large computational loads. Meanwhile the suboptimal algorithm of PDA (Bar-Shalom and Tse, 1975), is used here for Doppler ambiguity find the optimal solution for the current time. Based on the joint PDA (Roecker, 1994) frame, the joint integrated probabilistic data association (JIPDA) filter (Musicki and Evans, 2004), which concerns cluttered environment and track maintenance, also has been modified here to handle multitarget tracking.

The rest part of this paper is organized as follows: Section II builds on the state evolvement and measurement model, and reviews the UKF for this nonlinear systems. In section III, the Doppler ambiguity for a single target tracking problem is solved in different methods, and expended to the multitarget case in section IV. Section V is devoted to simulations evaluating the method performance in various simulation 
scenarios. Summary and conclusions are provided in section VI. 


\section{Chapter 2}

\section{OVERVIEW OF TARGET TRACKING}

\subsection{Filtering Algorithms}

In order to analyze and make inference about a dynamic system, two models are required. First, the system model, which describing the evolution of the state with time, is

$$
x_{k+1}=f_{k}\left(x_{k}\right)+v_{k}
$$

and second the measurement model which express the relation between the state and the noisy measurement is

$$
z_{k}=h_{k}\left(x_{k}\right)+\omega_{k}
$$

where $x_{k}$ is the state of the target and $z_{k}$ is the measurement vector at time $k$, and $v_{k} \omega_{k}$ are the Gaussian process noise and measurement noise, respectively. We will assume that these models are available. The probabilistic sate space formulation and 
the requirement for the updating of information on receipt of new measurements are ideally suited for the Bayesian approach. This provides a rigorous general framework for dynamic state estimation problems.

In the Bayesian approach to dynamic state estimation, one attempts to construction the posterior probability density function of the state based on all available information, including the prior information and all received measurement over time. Since this PDF embodies all available statistical information, it can be said to be the optimal solution of the estimation problem. IN principle, an optimal estimate of the state may be obtain form the PDF by maximum likelihood (ML) estimation. A recursive filtering approach means that the received data can be processed sequentially rather than as a batch so that it is not necessary to store the completed data set nor to reprocess existing data if a new measurement becomes available. This filter is composed of two steps: prediction and udpated. The prediction step used the system model to predict the state PDF to the next time scan. Since the state is usually subject to unknown disturbance, prediction generally translates, deforms or spreads the state PDF.

Assume that the required $\operatorname{PDF} p\left(x_{k} \mid Z^{k}\right)$ at time $k$, where $Z^{k}=\left[z_{1}, z_{2}, \ldots, z_{k}\right]$. The prediction step involves using the the system model to obtain the prior PFD fo the state at time $k+1$ and given by

$$
p\left(x_{k+1} \mid Z^{k}\right)=\int p\left(x_{k+1} \mid x_{k}\right) p\left(x_{k} \mid Z^{k}\right) d x_{k}
$$

The update operation uses the latest measurement to modify the prediction PFD. At time $k$, a measurement $z_{k}$ becomes available and will be used to update the prior 
via Bayes' rule

$$
p\left(x_{k+1} \mid Z^{k+1}\right)=\frac{p\left(z_{k+1} \mid x_{k+1}\right) p\left(x_{k+1} \mid Z^{k}\right)}{p\left(z_{k+1} \mid Z^{k}\right)}
$$

The above likelihood function is defined by the measurement model.

The above recursive propagation of the posterior density is only a theoretical solution by not practical. However, under certain assumptions, we can solve the the problem by Kalman Filter.

\subsection{Kalman Filter}

The Kalman filter assumes that the state and measurement model are linear and the initial state error and all noises, both process and measurement noises are Gaussian and hence, parameterized by a mean and covariance. Under the above assumptions, if $p\left(x_{k} \mid Z^{k}\right)$ is Gaussian, it can be proved that $p\left(x_{k+1} \mid Z^{k+1}\right)$ is also Gaussian.

Then, the state and measurement equations are given by

$$
\begin{aligned}
x_{k+1} & =F_{k} x_{k}+v_{k} \\
z_{k} & =H_{k} x_{k}+\omega_{k}
\end{aligned}
$$

If $F_{k}$ and $H_{k}$ are know matrices, $v_{k} \sim \mathcal{N}\left(0, Q_{k}\right)$ and $\omega_{k} \sim \mathcal{N}\left(0, R_{k}\right)$, the Kalman filter algorithm can then be viewed as the following recursive form:

$$
\begin{aligned}
p\left(x_{k} \mid Z^{k}\right) & =\mathcal{N}\left(x_{k} ; \hat{x}_{k \mid k}, P_{k \mid k}\right) \\
p\left(x_{k+1} \mid Z^{k}\right) & =\mathcal{N}\left(x_{k+1} ; \hat{x}_{k+1 \mid k}, P_{k+1 \mid k}\right) \\
p\left(x_{k+1} \mid Z^{k+1}\right) & =\mathcal{N}\left(x_{k+1} ; \hat{x}_{k+1 \mid k+1}, P_{k+1 \mid k+1}\right)
\end{aligned}
$$


where

$$
\begin{aligned}
\hat{x}_{k+1 \mid k} & =F_{k} \hat{x}_{k \mid k} \\
P_{k+1 \mid k} & =F_{k} P_{k \mid k} F_{k}^{T}+Q_{k} \\
\hat{x}_{k+1 \mid k+1} & =\hat{x}_{k+1 \mid k}+K_{k+1}\left(z_{k+1}-H_{k+1} \hat{x}_{k+1 \mid k}\right) \\
P_{k+1 \mid k} & =P_{k+1 \mid k}-K_{k+1} H_{k+1} P_{k+1 \mid k}
\end{aligned}
$$

with

$$
\begin{aligned}
S_{k+1} & =H_{k+1} P_{k+1 \mid k} H_{k+1}^{T}+R_{k+1} \\
K_{k+1} & =P_{k+1 \mid k} H_{k+1}^{T} S_{k+1}^{-1}
\end{aligned}
$$

In the above, $\mathcal{N}(x ; \hat{x}, P)$ means a Gaussian density with argument $x$, mean $\hat{x}$ and covariance $P$.

This is the optimal solution to the tracking problem under the above assumption. THe implication is that no algorithm can do better than a Kalman filter in this linear Gaussian environment.

In many situation of interest the assumption made above do not hold, where linear approximation or other methods are required to solve the problem.

\subsection{Extended Kalman Filter}

If the functions in (2.1) and (2.2) are nonlinear, then a local linearization of the equations could be a sufficient description of the nonlinearity. Local linearization of 
the above function are

$$
\begin{aligned}
& \hat{F}_{k}=\left.\frac{d f_{k}(x)}{d x}\right|_{x=\hat{x}_{k \mid k}} \\
& \hat{H}_{k}=\left.\frac{d h_{k}(x)}{d x}\right|_{x=\hat{x}_{k \mid k-1}}
\end{aligned}
$$

The EKF is based on the assumption that $p\left(x_{k} \mid Z^{k}\right)$ is approximation by a Gaussian. Then all the equation of the Kalman filter can be used with this approximation and the linearized function.

\subsection{Unscented Kalman Filter}

In general case, the state propagation and measurement models are nonlinear. With the explicit expression of the measurement and the Gaussian noise assumption, the UKF is able to handle the nonlinear tracking problem appropriately (Julier and Uhlmann, 2004). The most commonly used nonlinear filter, EKF only uses the first order terms of the Taylor series expansion of the nonlinear functions, and as a result often comes with major errors in the estimated statistics of the posterior distributions of the states, especially in the highly nonlinear or even non-differentiable case. Unlike the EKF, the UKF uses true models rather than linearly approximate the nonlinear process and observation model. However, the UKF is restricted to the Gaussian case and is specified by using a minimal set of deterministically chosen samples, which can completely capture the true mean and covariance of the Gaussian random variable. Even when propagated by the true nonlinear model, the posterior mean and covariance can still be accurately estimated, while errors only exist in the third or higher order. To implement UKF, the unscented transformation is applied in (Julier 
and Uhlmann, 2004) (Wan and Van Der Merwe, 2000).

Assume state $x_{k-1}$ has mean $\hat{x}_{k-1}$ and covariance $P_{x}$. To calculate the first two moments of $\hat{x}_{k}$ using the UT, the following procedure is undertaken. First, a set of $2 n_{x}+1$ weighted samples $\mathcal{S}_{i}=\left\{W_{i}, \mathcal{X}_{i}\right\}$, called sigma points are generated, where $n_{x}$ is the dimension of $\hat{x}_{k}$ and $i$ is the index of such points. Those sigma points are deterministically chosen so that they completely capture the true mean and covariance of the prior random variable $\mathbf{x}$. However, within the system of Gaussian process noise $v$ and measurement noise $\omega$, the state random variable is redefined as $x^{a}=[x ; v ; \omega]$. The sigma point selection scheme is applied to this new state random variable to calculate the corresponding sigma matrix, $\mathcal{X}^{a}$ (the set of all sigma points). The complete UKF algorithm is given as follows:

1. Initialization: at time $k=0$

$$
\begin{aligned}
& \hat{x}=E\left[x_{0}\right] \\
& P_{0}=E\left[\left(x_{0}-\hat{x}\right)\left(x_{0}-\hat{x}\right)^{T}\right] \\
& \hat{x}_{0}^{a}=E\left[x_{0}^{a}\right]=\left[\hat{x}_{0}^{T}, 0_{1 \times n_{v}}, 0_{1 \times n_{w}}\right]^{T} \\
& P_{0}^{a}=E\left[\left(x_{0}^{a}-\hat{x}^{a}\right)\left(x_{0}^{a}-\hat{x}^{a}\right)^{T}\right]=\left[\begin{array}{ccc}
P_{0} & 0 & 0 \\
0 & Q & 0 \\
0 & 0 & R
\end{array}\right]
\end{aligned}
$$

2. For $k \in\{1, \ldots, \infty\}$ 
- Calculate sigma points

$$
\mathcal{X}_{k-1}^{a}=\left[\hat{x}_{k-1}^{a}, \hat{x}_{k-1}^{a} \pm \sqrt{\left(n_{a}+\lambda\right) P_{k-1}^{a}}\right]
$$

- Prediction

$$
\begin{aligned}
\mathcal{X}_{k \mid k-1}^{x} & =f\left(\mathcal{X}_{k-1}^{x}, \mathcal{X}_{k-1}^{v}\right) \\
\hat{x}_{k \mid k-1} & =\sum_{i=0}^{2 n_{a}} W_{i} \mathcal{X}_{i, t \mid t-1}^{x} \\
P_{t \mid t-1} & =\sum_{i=0}^{2 n_{a}} W_{i}\left[\mathcal{X}_{i, k \mid k-1}^{x}-\hat{x}_{k \mid k-1}\right]\left[\mathcal{X}_{i, k \mid k-1}^{x}-\hat{x}_{k \mid k-1}\right] T \\
\mathcal{Z}_{k \mid k-1} & =h\left(\mathcal{X}_{k \mid k-1}^{x}, \mathcal{X}_{k \mid k-1}^{w}\right) \\
\hat{z}_{k \mid k-1} & =\sum_{i=0}^{2 n_{a}} W_{i} \mathcal{Z}_{i, k \mid k-1}
\end{aligned}
$$

- Measurement update equation

$$
\begin{aligned}
P_{z_{k} z_{k}} & =\sum_{i=0}^{2 n_{a}} W_{i}\left[\mathcal{Z}_{i, k \mid k-1}-\hat{z}_{k \mid k-1}\right]\left[\mathcal{Z}_{i, k \mid k-1}-\hat{z}_{k \mid k-1}\right]^{T} \\
P_{x_{k} z_{k}} & =\sum_{i=0}^{2 n_{a}} W_{i}\left[\mathcal{X}_{i, k \mid k-1}^{x}-\hat{x}_{k \mid k-1}\right]\left[\mathcal{Z}_{i, k \mid k-1}-\hat{x}_{k \mid k-1}\right]^{T} \\
K_{t} & =P_{x_{k} z_{k}} P_{z_{k} z_{k}}^{-1} \\
\hat{x}_{k} & =\hat{x}_{k \mid k-1}+K_{k}\left(z_{k}-\hat{z}_{k \mid k-1}\right) \\
P_{k} & =P_{k \mid k-1}-K_{k} P_{z_{k} z_{k}} K_{k}^{T}
\end{aligned}
$$

where, $x^{a}=\left[x^{T}, x^{T}, \omega^{T}\right], \mathcal{X}^{a}=\left[\left(\mathcal{X}^{x}\right)^{T},\left(\mathcal{X}^{v}\right)^{T},\left(\mathcal{X}^{w}\right)^{T}\right], \lambda$ is the composite scaling parameter, $n_{a}=n_{x}+n_{v}+n_{w}$, and the weight $W_{i}, i=0, \ldots, 2 n_{a}$ is 
calculated by

$$
\begin{aligned}
& W_{0}=\lambda /\left(n_{a}+\lambda\right) \\
& W_{i}=1 / 2\left(n_{a}+\lambda\right), i=1, \ldots, 2 n_{a}
\end{aligned}
$$




\section{Chapter 3}

\section{PROBLEM STATEMENT}

At time $t_{1}, t_{2}, \ldots$ measurements $z_{1}, z_{2}, \ldots$ are provided, each belonging to the measurement space $\mathbb{Z}$. The target moves through a state space $\mathbb{X}$ and for time $t_{k}$ has state $\mathbf{x}_{k}$. The task is to estimate the state with the knowledge of the sequence of radar measurement.

\subsection{Target State Propagation}

The target state in the 2D Cartesian space is composed of the horizontal position, vertical position, horizontal velocity and vertical velocity, which is

$$
\mathbf{x}_{k}=[x, y, \dot{x}, \dot{y}]^{\prime}
$$

Generally, the nonlinear state evolution model is given as:

$$
\mathbf{x}_{k+1}=F_{k}\left(\mathbf{x}_{k}, v_{k}\right)
$$


with the process noise $v_{k}$ a zero-mean white acceleration sequence. For simplicity of the mathematics deduction, a linear time invariant system with constant velocity model and the fixed sampling period $T$ is considered here.

$$
\mathbf{x}_{k+1}=F_{k} \mathbf{x}_{k}+\Gamma_{k} v_{k}
$$

observe that the result algorithm also works for the nonlinear system, or other more complicated scenario, like multiple model (MM) or interacting multiple model(IMM).

The propagation matrix is taken as

$$
F_{k}=F=\left[\begin{array}{cccc}
1 & 0 & T & 0 \\
0 & 1 & 0 & T \\
0 & 0 & 1 & 0 \\
0 & 0 & 0 & 1
\end{array}\right]
$$

and the noise gain is

$$
\Gamma_{k}=\Gamma=\left[\begin{array}{cc}
\frac{1}{2} T^{2} & 0 \\
0 & \frac{1}{2} T^{2} \\
T & 0 \\
0 & T
\end{array}\right]
$$

The covariance of the process noise multiplied by the gain, $\Gamma v_{k}$, is

$$
Q=E\left[\Gamma v_{k} v_{k}^{\prime} \Gamma^{\prime}\right]=\left[\begin{array}{cccc}
\frac{1}{4} T^{4} & 0 & \frac{1}{2} T^{3} & 0 \\
0 & \frac{1}{4} T^{4} & 0 & \frac{1}{2} T^{3} \\
\frac{1}{2} T^{3} & 0 & T^{2} & 0 \\
0 & \frac{1}{2} T^{3} & 0 & T^{2}
\end{array}\right] \sigma_{v}^{2}
$$


where

$$
\sigma_{v}^{2}=E\left[v_{k, x}^{2}\right]=E\left[v_{k, y}^{2}\right]
$$

is the acceleration noise along each axis.

\subsection{Target Generated Measurements}

The investigation provided here focused on the combination of two kinds of measurements in two space variants: position measurement provided in spherical coordinates and Doppler measurements. And the observation of target are modeled by an additive noise process

$$
z_{k}=h\left(\mathbf{x}_{k}\right)+w_{k}
$$

where $h$ is measurement model composed of following parts

$$
h\left(\mathbf{x}_{k}\right)=\left[\begin{array}{c}
r\left(\mathbf{x}_{k}\right) \\
\theta\left(\mathbf{x}_{k}\right) \\
\dot{r}\left(\mathbf{x}_{k}\right) \text { or } \Delta \dot{r}\left(\mathbf{x}_{k}\right)
\end{array}\right]
$$

where the range measurement $r$

$$
r\left(\mathbf{x}_{k}\right)=\sqrt{x^{2}+y^{2}}
$$

bearing measurement $\theta$

$$
\theta\left(\mathbf{x}_{k}\right)=\arctan (y / x)
$$


and Doppler measurement $\dot{r}$ without ambiguity or $\Delta \dot{r}$ with ambiguity is

$$
\begin{aligned}
\dot{r}\left(\mathrm{x}_{k}\right) & =\frac{d r}{d t} \\
& =\left(\frac{x \dot{x}+y \dot{y}}{\sqrt{x^{2}+y^{2}}}\right) \\
\Delta \dot{r} & =\bmod \left(\dot{r}, v_{f}\right)
\end{aligned}
$$

$v_{f}$ is a given constant named first blind velocity which is determined by the PRF as

$$
v_{f}=\frac{1}{2} \lambda \times P R F
$$

where $\lambda$ is the wavelength of the radar waves.

The measurement noise $w_{k}$ is a white, zero mean gaussian random variable with covariance

$$
R=\left[\begin{array}{ccc}
\sigma_{r}^{2} & 0 & \rho \sigma_{r} \sigma_{\dot{r}} \\
0 & \sigma_{\theta}^{2} & 0 \\
\rho \sigma_{r} \sigma_{\dot{r}} & 0 & \sigma_{\dot{r}}^{2}
\end{array}\right]
$$

where $\sigma_{r}, \sigma_{\theta}$ and $\sigma_{\dot{r}}\left(\sigma_{\Delta \dot{r}}=\sigma_{\dot{r}}\right)$ are the standard deviation of the additive white noise for the range, bearing and Doppler measurement, respectively. According to (Bar-Shalom, 2000), there is a strong negative correlation between the range measurement error and Doppler measurement error, e.g., $\rho=-0.9$. However, here each measurement is independent of each other, thus $\rho=0$. 


\section{Chapter 4}

\section{SINGLE TARGET TRACKING WITH DOPPLER AMBIGUITY}

\subsection{UKF with Doppler Ambiguity}

The measurement can be written as

$$
z=\left[z_{p}, z_{d}\right]^{\prime}
$$

where $z_{p}=[r, \theta]$ is the position measurement and $z_{d}$ is the Doppler measurement. Two types of Doppler measurements are considered here: Unambiguous Doppler $z_{d}^{U}$ and Ambiguous Doppler $z_{d}^{A}$. Moreover, for a certain target, two types of Doppler measurements can be written as

$$
z_{d}^{U}=z_{d}^{A}+n_{d} v_{f}
$$


where $n_{d}$ is noted as Doppler order here.

Assume that in Fig. 4.1 the circles are the sigma points of $z_{d}^{U}$, which hold the first and second order of Gaussian variable's statistic property, the mean and covariance, during the unscented transformation. However, when the implement of UKF with Doppler ambiguity, the distribution of cross sigma points ( the components of $z_{d}^{U}$ ) might vary because of the discontinuity of the mod function, illustrated as the following figure :
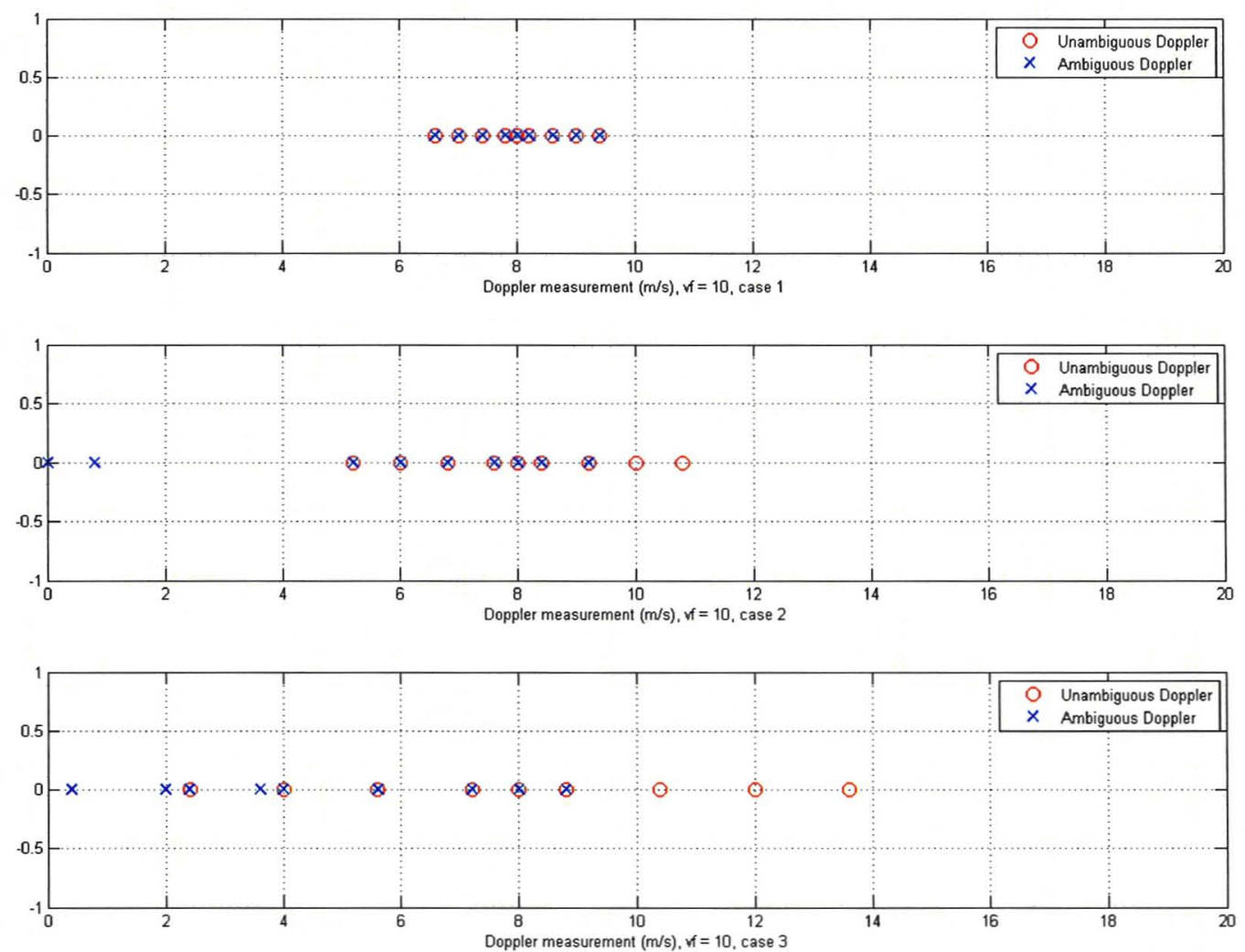

Figure 4.1: Examples of Sigma Points Distribution

For the first case, all the sigma points for the ambiguous Doppler measurement 
$z_{i, d}^{U}$ have the same value of Doppler order, thus for each sigma point $i, n_{i, d}=n_{0, d}$. Thus, compared to the unambiguous Doppler $z_{i, d}^{U}$, it holds that

$$
\overline{\mathrm{z}}_{t \mid t-1}^{A}=\sum_{i=0}^{2 n_{a}} W_{i}\left(z_{i, t \mid t-1}^{U}-n_{0, d} v_{f}\right)=\overline{\mathbf{z}}_{t \mid t-1}^{U}-n_{0, d} v_{f}
$$

and

$$
P_{z_{t}^{A} z_{t}^{4}}=\sum_{i=0}^{2 n_{a}} W_{i}\left[z_{i, t \mid t-1}^{U}-\overline{\mathbf{z}}_{t \mid t-1}^{U}\right]^{2}=P_{z_{t}^{U} z_{t}^{U}}
$$

where the mean shifts by $n_{0, d} v_{f}$ and the covariance conserves. Therefore, in this case the UKF can be implemented directly with ambiguous Doppler measurement.

In the second case, there exists at least one sigma point that $n_{i, d} \neq n_{0, d}$. It can be seen that the distribution of the $z_{i, d}^{A}$ is different from $z_{i, d}^{U}$ because of the "wrapped" points. The mean and covariance of $z_{i, d}^{A}$ can be written as

$$
\begin{aligned}
\overline{\mathbf{z}}_{t \mid t-1}^{A}= & \sum_{i=0}^{2 n_{a}} W_{i}\left(\mathcal{Z}_{i, t \mid t-1}^{U}-n_{i, d} v_{f}\right) \\
= & \overline{\mathbf{z}}_{t \mid t-1}^{U}-n_{0, d} v_{f}-\sum_{i, n_{i, d}=n_{d, 0}+1} W_{i} M \\
= & \overline{\mathbf{z}}_{t \mid t-1}^{U}-n_{0, d} v_{f}-C \\
P_{z_{t}^{A} z_{t}^{A}}= & \sum_{i=0}^{2 n_{a}} W_{i}\left[\mathcal{Z}_{i, t \mid t-1}^{U}-\overline{\mathbf{z}}_{t \mid t-1}^{U}-n_{d, i} v_{f}+n_{0, d} v_{f}+C\right]^{2} \\
= & P_{z_{t}^{U} z_{t}^{U}}-C^{2}+v_{f} C+ \\
& \sum_{i, n_{i}=n+1} 2 v_{f} W_{i}\left[\mathcal{Z}_{i, t \mid t-1}^{U}-\overline{\mathbf{z}}_{t \mid t-1}^{U}-n_{i} v_{f}-n_{0, d} v_{f}-C\right]
\end{aligned}
$$

where $C=\sum_{i, n_{i, d}=n_{d, 0}+1} W_{i} M$. Generally, the mean and covariance have been corrupted for "wrapping" purpose.

To solve this problem, assume that the range of the $z_{i, d}^{U}$ for all $i$ is short compared 
to the value of $v_{f}$, so that it is reasonable to hold that

$$
\max _{i}\left|z_{i, d}^{U}-z_{0, d}^{U}\right| \leq v_{f} / 2
$$

Therefore, in order to make sure that all the points have the same order $n_{0}$ under the previous assumption, the "wrapped" points can be modified by shifting them closer to center point $z_{0, d}^{A}$ : For $i>0$

- if $\mathcal{Z}_{i, t \mid t-1}^{A}-\mathcal{Z}_{0, t \mid t-1}^{U}>v_{f} / 2$, then $\mathcal{Z}_{i, t \mid t-1}^{A}=\mathcal{Z}_{i, t \mid t-1}^{A}-v_{f}$

- if $\mathcal{Z}_{i, t \mid t-1}^{A}-\mathcal{Z}_{0, t \mid t-1}^{U}<-v_{f} / 2$, then $\mathcal{Z}_{i, t \mid t-1}^{A}=\mathcal{Z}_{i, t \mid t-1}^{A}+v_{f}$

Observe that after the shifting, Doppler measurement $z_{i, d}^{A}$ for each sigma point is no longer ranged in $[0, M]$ but with identical Doppler order.

In the third case,

$$
\max _{i}\left|z_{i, d}^{U}-z_{0, d}^{U}\right|>v_{f} / 2
$$

It is impossible to determine shifting methods for the wrapped points, because those points are closer to the center point, with assigned a false Doppler order rather than the correct one. However, this type of widespread case rarely happen due to the high accuracy of the Doppler measurement.

The innovation of measurement is also an ambiguous problem. Assume the predict mean $\overline{\mathbf{z}}_{d}^{A}$ and covariance is correct, with the receiving measurement $z_{d}^{A}$, the innovation can be written as

$$
\begin{aligned}
\nu^{U} & =z^{A}+n_{d} v_{f}-\overline{\mathbf{z}}_{t \mid t-1}^{A}-n_{0, d} v_{f} \\
& =\nu^{A}-\Delta n_{d} v_{f}
\end{aligned}
$$


where $\nu^{A}$ and $\nu^{U}$ denote the innovation of unambiguous Doppler and ambiguous Doppler, respectively. Observe that only $\nu^{U}$ is the correct range rate innovation. Because of the difference between the Doppler order of the prediction and true measurement, the innovation $\nu^{A}$ could be corrupted by $\Delta n_{d} v_{f}$, where $\Delta n_{d}$ is unknown difference. To solve this unknown ambiguity, under previous accuracy assumption, it is reasonable to choose the closest candidate value of $z_{d}^{U}$ to the prediction $\bar{z}_{t \mid t-1}^{U}$ by ML estimation, as our innovation in Kalman update. It is implemented as

$$
\begin{aligned}
\Delta n_{d}^{*} & =\arg \min _{\Delta n_{d}}\left|\nu^{U}\right|=\arg \min _{\Delta n}\left|\nu^{A}-\Delta n_{d} v_{f}\right| \\
\nu^{U *} & =\nu^{A}-\Delta n_{d}^{*} v_{f}
\end{aligned}
$$

\subsection{MHT with Doppler ambiguity}

In this section, it is assumed that: 1) The measurement-to-track association is done.

2) There is only one target of interest and, modeled by (3.5). 3) The track has been well initialized. 4) One associated measurement, with Doppler ambiguity $z_{k}^{A}=$ $\left[z_{p, k}, z_{d, k}^{A}\right]^{\prime}$ is received at time $k$, short for $z_{k}$ modeled by (3.9).

Now, based on the measurement model, the receiving measurement is composed of position $z_{p, k}$ and ambiguous Doppler measurement $z_{d, k}$. According to (3.13), assume that the Doppler order takes the candidate value form the Doppler order set $n_{d}=$ $\left\{-n_{\max }, \ldots,-1,0,1, \ldots, n_{\max }\right\}$ or written as $n_{d}=\left\{n_{i}\right\}_{i=1}^{N}$ for convenience, where $\mathrm{i}$ the index of the chosen Doppler order in the set and $N=2_{n_{\max }}+1$. And the value of Doppler order is bounded by the maximum velocity $v_{\max }$ that $\left|v_{\max }\right| \leq n_{\max } v_{f}$.

In the section of UKF tracking, $n_{d}$ is determined via ML estimation, which could be incorrect in some certain case. In the following methods, MHT and PDA, which 
used to be the algorithm solving the ambiguity of measurement-to-track association, are applied to solve the Doppler ambiguity problem (unknown Doppler order).

The MHT algorithm is presented to deal with the measurement-to-track association in multitarget tracking (Reid, 1979). The Key idea of MHT is exhaustively searching all possible association events from time 1 to time $\mathrm{k}$ and find the one with greatest probability. Instead of measurement-to-track association ambiguity, the MHT algorithm is applied to solve the Doppler ambiguity, or the value of the Doppler order of each measurement in this paper.

Let a cumulative event, a fix sequence of Doppler order through time $k$ be

$$
\Theta^{k, j}=\left\{\Theta^{k-1, s}, \theta(k)\right\}
$$

This cumulative event is made of a parent event through $k-1$ and the offspring event or current ambiguity event, which allocates a feasible value of Doppler order for the current measurement, where the current ambiguity event

$$
\theta_{i}(k)=\left\{n_{d, k}=i, i \in\{0, \ldots, N\}\right\}
$$

means the Doppler order for measurement $z_{k}$ is equal to $i$ in event $\theta_{i}(k)$.

The set $\Theta^{k}=\left\{\Theta^{k, j}\right\}_{j}$ contains all the possible ambiguity hypotheses. Each hypotheses in this set provides a fixed sequence of Doppler order from time 1 to $k$. The MHT requires an exhaustive search, which means all the hypotheses are generated and evaluated in order to find the most probable hypothesis. The representation of these hypotheses is done using tree data structure. 
With Bayes' formula, the conditional probability of cumulative event is

$$
\begin{aligned}
P\left\{\Theta^{k, j} \mid Z^{k}\right\} & =P\left\{\theta(k), \Theta^{k-1, s} \mid z_{k}, Z^{k-1}\right\} \\
& =\frac{P\left\{z_{k}, \theta(k) \mid \Theta^{k-1, s}, Z^{k-1}\right\} P\left\{\Theta^{k-1, s} \mid Z^{k-1}\right\}}{P\left\{z_{k} \mid Z^{k-1}\right\}} \\
& =\frac{1}{c_{0}} P\left\{z_{k}, \theta(k) \mid \Theta^{k-1, s}, Z^{k-1}\right\} P\left\{\Theta^{k-1, s} \mid Z^{k-1}\right\} \\
& =\frac{1}{c_{0}} P\left\{z_{k} \mid \theta(k), \Theta^{k-1, s}, Z^{k-1}\right\} P\left\{\theta(k) \mid \Theta^{k-1, s}, Z^{k-1}\right\} P\left\{\Theta^{k-1, s} \mid Z^{k}\left(4^{1} .\right\} 5\right)
\end{aligned}
$$

where $\frac{1}{c_{0}}$ is the normalization factor. The second item on the right-hand side (RHS) can be written as $P\left\{z_{k} \mid \theta(k), Z^{k-1}\right\}$. Assume that $\theta(k)=\theta_{i}(k)=\left\{n_{k}=i\right\}$, then the ambiguity of Doppler measurement is solved by

$$
P\left\{z_{k} \mid \theta_{i}(k), Z^{k-1}\right\}=P\left\{z_{k}^{U} \mid \theta_{i}(k), Z^{k-1}\right\}
$$

where $z_{d, t}^{U}=z_{d, t}^{A}+i M$. This item is the likelihood of the unambiguous measurement.

The third item on the RHS in (4.15) is the prior probability of ambiguity event. A uniform distribution is used here, thus

$$
P\left\{\theta(k) \mid \Theta^{k-1, s}, Z^{k-1}\right\}=1 / N
$$

The last item on the RHS in (4.15) is the parent's hypothesis probability.

After all, equation (4.15) can be written as

$$
P\left\{\Theta^{k, j} \mid Z^{k}\right\}=\frac{1}{c} P\left\{z_{k}^{U} \mid \theta(k), Z^{k-1}\right\} P\left\{\Theta^{k-1, s} \mid Z^{k-1}\right\}
$$


Based on above analysis, the MHT approach considers both the association of sequences of Doppler order and evaluation of the probabilities of all ambiguity hypotheses. One the other hand, MHT will result in a complexity increasing exponentially with time. Appropriate methods, like valid gating and pruning of low probability hypotheses, are applied to constrain the number of hypotheses. Fig. 4.2 provides an example of how to evolve and manage hypotheses.

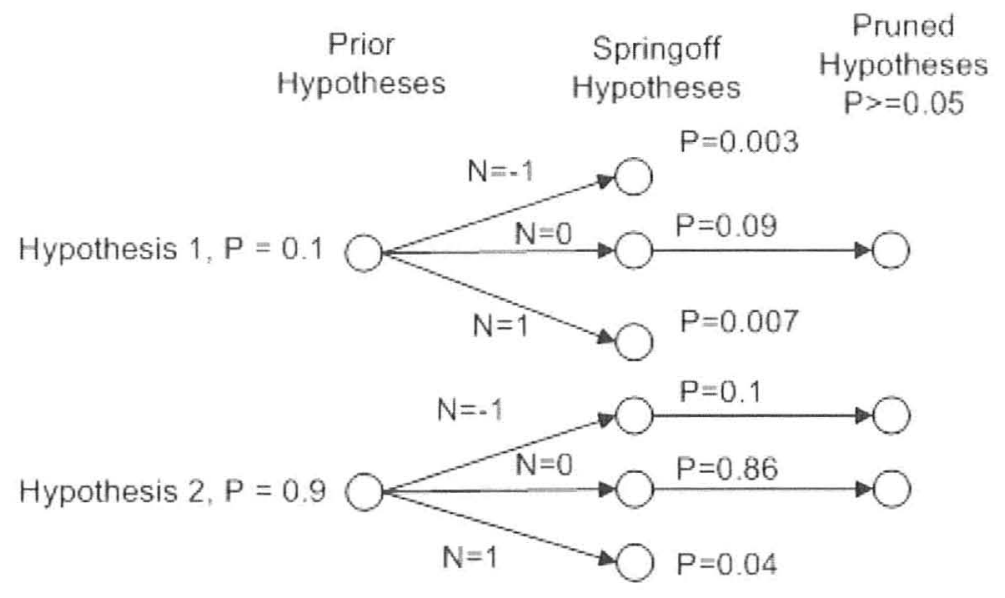

Figure 4.2: Example of Evolving and Managing Hypotheses

In this figure, new spring-off hypotheses are generated based on two existing parent hypotheses. The probability of each spring-off hypothesis is calculated by equation (4.18). Then all these hypotheses are managed by pruning the ones with low probability and the survived ones are unified and propagated into the next run.

\subsection{PDA with Doppler Ambiguity}

MHT is considered as the optimal algorithm to solve ambiguity problems. However, it is limited by the computational load because of exponentially increasing number 
of hypotheses. A suboptimal method, Probabilistic Data Association ( PDA) (BarShalom and Tse, 1975), is used here as suboptimal solution for Doppler ambiguity in single target tracking.

In addition to assumptions in previous section, assume that the past information about the target is summarized as the state estimate $\hat{x}_{k-1 \mid k-1}=E\left\{x_{k-1} \mid Z^{k-1}\right\}$ and the associated covariance $P(k-1)$. Notice that this assumption is only valid for PDA based tracking. Importantly, this assumption makes it possible to carry out an association w.r.t the latest measurements only, comapring to association of all history measurements in MHT.

The estimation is weighted sum of each possible ambiguity events. With the total probability theorem w.r.t the current measurement association events, the conditional mean of the state at time $k$ can be written as

$$
\begin{aligned}
\hat{x}_{k \mid k} & =\sum_{g=1}^{m_{k}} E\left[x_{k} \mid a_{g, k}, Z^{k}\right] P\left\{a_{g, k} \mid Z^{k}\right\} \\
& =\sum_{g=1}^{m_{k}} \hat{x}^{g, k \mid k} \beta_{g, k}
\end{aligned}
$$

where $\hat{x}^{g, k \mid k}$ is the updated state conditioned on the event that the $g$-th candidate measurement is correct and

$$
\beta_{g, k} \triangleq P\left\{a_{g, k} \mid Z^{k}\right\}
$$

is the conditional probability of this ambiguity event, or the association probability. 


\subsubsection{The standard PDA Update}

Combining the equation above yields the state update equation of the PDAF

$$
\hat{x}_{k \mid k}=\hat{x}_{k \mid k-1}+W_{k} \nu_{k}
$$

where the combined innovation is

$$
\nu_{k}=\sum_{i=1}^{m_{k}} \beta_{i}(k) \nu_{i}(k)
$$

The covariance associated with the updated state is

$$
P(k \mid k)=P^{c}(k \mid k)+\tilde{P}(k)
$$

where the covariance of the state updated with the correct measurement is

$$
P^{c}(k \mid k)=P(k \mid k-1)-W_{k} S_{k} W_{k}^{\prime}
$$

and the spread of the innovations term is

$$
\tilde{P}(k) \triangleq W_{k}\left[\sum_{i=1}^{m_{k}} \beta_{i, k} \nu_{i, k} \nu_{i, k}^{\prime}-\nu_{k} \nu_{k}^{\prime}\right] W_{k}
$$

\subsubsection{Probability of Ambiguity Events}

To evaluate the association probabilities, it is written as

$$
\beta_{i}(k)=p\left\{a_{i, k} \mid Z^{k}\right\}=\frac{1}{c} P\left\{Z(k) \mid a_{i, k}, m_{k}, Z^{k-1}\right\} P\left\{a_{i}(k) \mid m_{k}, Z^{k-1}\right\}
$$


where $P\left\{Z(k) \mid a_{i, k}, m_{k}, Z^{k-1}\right\}$, the joint density of the validated measurements conditioned on $a_{i, k}$ is the product of the Gaussian pdf of the correct measurement and the pdf of the incorrect measurement, which is assumed uniform in the validation region. 


\section{Chapter 5}

\section{MULTITARGET TRACKING WITH DOPPLER AMBIGUITY}

\subsection{Track-Measurement-Doppler Ambiguity Asso- ciation}

The data association yields decisions as to which of the received measurements should be used to update each track. The assignment is formulated as a constraint optimization problem, where the cost function to be minimized is a combined negative loglikelihood ratio (LLR) evaluated using the results from the state estimator. Moreover, to deal with the Doppler ambiguity, not only the measurement-to-track association, but also the measurement-to-Doppler order association must be performed. Modified assignment formulation with ambiguous Doppler measurement are proposed to resolve the considered data association problem.

For already established tracks, the problem is to associate the measurement to the 
targets and doppler ambiguity order. To present the assignment problem, the tree diagram in Fig.5.1 provides one example of assignment shown as follow.

In Fig.5.1, dummy target or dummy measurement indicates a false track or a miss detection, respectively. The connection between point in figure means each measurement is assigned to one target and one fixed Doppler order. A more straightforward illustration for the same assignment is shown in Fig.5.2. In this figure the Doppler order is decoupled for each measurement, which is independent of each other in the assignment step. In this case, a 3-D assignment will be presented.

$$
\text { Target Measurement Doppler Order }
$$

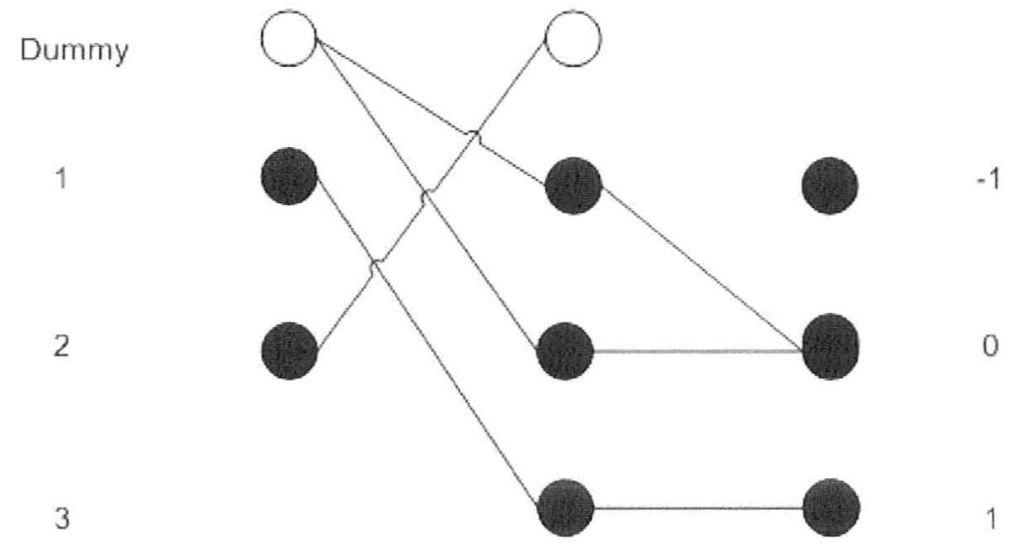

Figure 5.1: Example of One Assignment

Define a binary assignment variable $a(k, m, t, n)$

$a(k, m, t, n)= \begin{cases}1, & \text { measurement } \mathrm{m} \text { is associated with track } \mathrm{t}, \text { with Doppler ambiguity order o } \\ 0, & \text { otherwise. }\end{cases}$

where $t$ is the index of the established tracks at time $k, t=0,1, \ldots, T(k), m$ is the index of the observed measurements at time $k, m=0,1, \ldots, M(k)$, and $n$ is the 


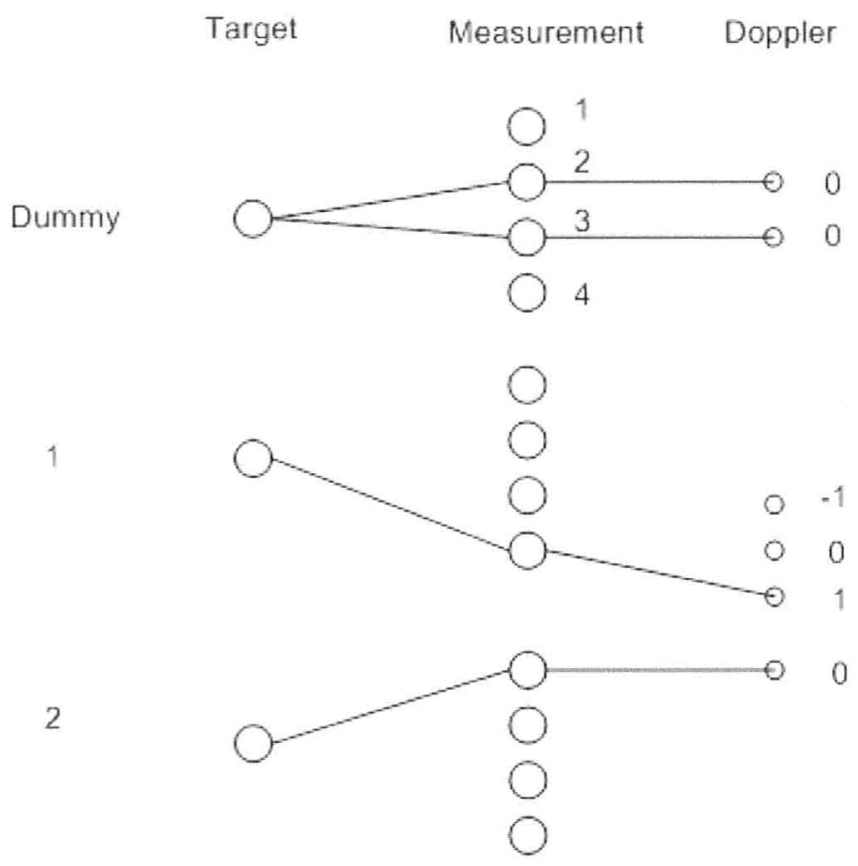

Figure 5.2: Assignment Problem

Doppler ambiguity order, $n=1, \ldots, N$. For the dummy measurement, the Doppler ambiguity order is set to 0 by default.

The assignment has to satisfy the following constraints

$$
\begin{aligned}
\sum_{t=0}^{T} \sum_{n=1}^{N} a(k, m, t, n) & =1, m=1, \ldots, M(k) \\
\sum_{m=0}^{M} \sum_{n=1}^{N} a(k, m, t, n) & =1, t=0,1, \ldots, T(k) \\
\sum_{n=1}^{N} a(k, m, t, n) & \leq 1, t=0,1, \ldots, T, m=0,1, \ldots, M(k)
\end{aligned}
$$

The first constraint equation is that one observed measurement can only assigned to one track. The second constraint equation is that one established track can be associated with one measurement. The third constraint equation is that for one measurement, at most one Doppler ambiguity order is valid. However, it can be 
deduced from the first two constraints.

Define the global assignment variable for current time $k$

$$
\mathbf{a}(k)=\bigcap_{m} a(k, m, t, n), \text { constrained by }(5.2),(5.3)
$$

is one possible assignments for all current measurement.

\subsection{MHT Algorithm}

The optimal solution is to find the best sequence of assignment through time $k$. Define a joint cumulative event (set of association histories ) at $k$

$$
\mathbf{A}^{k, l}=\left\{\mathbf{A}^{k-1, s}, \mathbf{a}(k)\right\}
$$

which is made up of a parent event through $k-1$ and the current association event defined in [5.5]. With respect to a certain current association $\mathbf{a}(k)$, the following notations are defined for the purpose of further analysis. The detection event where measurement is assigned with established tracks for current association $\mathbf{a}(k)$ is

$$
\mathbf{a}_{T}(k)=\bigcap_{m, t \neq 0} a(k, m, t, n), a(k, m, t, n) \in \mathbf{a}(k)
$$

where current measurement $m$ is associated with existing track $t$. And the number of associated measurements is $N_{D}$. The new target or false alarm event where measurement is assigned with new target or for current association $\mathbf{a}(k)$ is

$$
\mathbf{a}_{N F}(k)=\bigcap_{m, t=0} a(k, m, t, n), a(k, m, t, n) \in \mathbf{a}(k)
$$


where measurement $m$ is associated with dummy track. The number of measurement associated to false alarm or new target is $N_{N F}$. Notice that the observed measurement is either generated by the existing target or by false alarm, which holds $N_{D}+N_{N F}=$ $m(k)$. On the other hand, the new target is not distinguished from false alarms in our association, which is a little different from the standard MHT. The purpose of this unification is to decrease the number of association events and make the MHT more practical.

The following part of this section is how to calculate the probability of each hypotheses based on the standard MHT. With Bayes' rules, the joint association event probabilities are

$$
\begin{aligned}
P\left\{\mathbf{A}^{k, l} \mid Z^{k}\right\} & =P\left\{\mathbf{A}^{k-1, s}, \mathbf{a}(k) \mid Z(k), Z^{k-1}\right\} \\
& =\frac{1}{c} p\left[Z(k) \mid \mathbf{a}(k), \mathbf{A}^{k-1, s}, Z^{k-1}\right] P\left\{\mathbf{a}(k) \mid \mathbf{A}^{k-1, s}, Z^{k-1}\right\} P\left\{\mathbf{A}^{k-1, s}\right\}(5 .
\end{aligned}
$$

where $c$ is the normalization constant that

$$
\sum_{l}^{N_{H}} P\left\{\mathbf{A}^{k, l}\right\}=1
$$

$N_{H}$ is the total number of hypotheses at time $\mathrm{k}, m(k)$ is number of current measurements and $P\left\{\mathrm{~A}^{k-1, s}\right\}$ on the RHS of the above equation is the probability of parent hypothesis. The other two terms on the RHS, $p\left[Z(k) \mid \mathbf{a}(k), \mathbf{A}^{k-1, s}, Z^{k-1}\right]$ and $P\left\{\mathbf{a}(k) \mid \mathbf{A}^{k-1, s}, Z^{k-1}\right\}$, will be evaluated as follows.

If the $i$ th measurement $z_{i}$ associated with track $t_{i}$, the pdf of $z_{i}$ is noted as $f_{t_{i}(\cdot)}$, which is the innovation pdf from a standard KF. Observe that according to (4.16), the ambiguity has been solved given the condition of a fixed hypothesis $\mathbf{a}(k)$. And 
for a false alarm or new target, the pdf is uniform in the surveillance volume $V$, i.e., $V^{-1}$.

The first term on the RHS in (5.14) is the likelihood function of the measurements $Z(k)$, given the association hypothesis a $(k)$. Thus

$$
\begin{aligned}
p\left[Z(k) \mid \mathbf{a}(k), \mathbf{A}^{k-1, s}, Z^{k-1}\right] & =\prod_{i \in \mathbf{a}_{T}(k)}^{m(k)} f_{t_{i}}\left(z_{i, k}^{U}\right) \prod_{i \in \mathbf{a}_{N F}(k)}^{m(k)} V^{-1} \\
& =V^{-N_{N F}} \prod_{i \in \mathbf{a}_{T}(k)}^{m(k)} f_{t_{i}}\left(z_{i, k}^{U}\right)
\end{aligned}
$$

Notice that the likelihood of the $z_{i, k}^{U}$ is used here. Therefore, instead of the position measurement only, Doppler measurement also contributes to the probability of each hypothesis, which helps to make a better association. For example in Fig.5.3, two measurements, $z_{1}$ and $z_{2}$, fall into the valid gate of one track $t_{1}$. With only the position measurement, the probabilities of associated track $t_{1}$ with measurement $z_{1}$ or $z_{2}$ are almost the same. While taking Doppler measurements into consideration, track $t_{1}$ would be more likely to associate with $z_{1}$ rather than $z_{2}$.

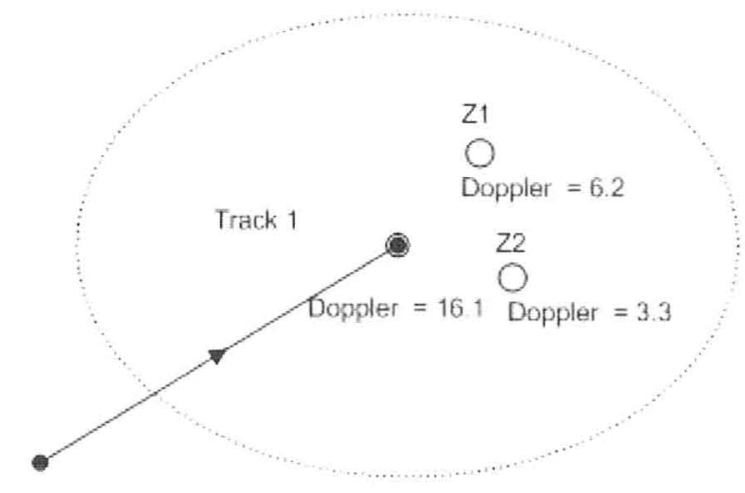

Figure 5.3: Example of Association with Doppler Measurement 
The second term on the RHS in (5.14) is the probability of a current dataassociation hypothesis given the prior hypothesis $\mathbf{A}^{k-1, s}$, which can be written as

$$
\begin{aligned}
P\left\{\mathbf{a}(k) \mid \mathbf{A}^{k-1, s}, Z^{k-1}\right\} & =P\left\{\mathbf{a}^{0}(k) \mathbf{a}^{d}(k) \mid \mathbf{A}^{k-1, s}, Z^{k-1}\right\} \\
& =P\left\{\mathbf{a}^{0}(k) \mid \mathbf{A}^{k-1, s}, Z^{k-1}\right\} P\left\{\mathbf{a}^{d}(k) \mid \mathbf{a}^{0}(k), \mathbf{A}^{k-1, s}, Z^{k-1}(3) .12\right)
\end{aligned}
$$

Here the current assignment variable is broken into two parts:

1. Measurement-to-Track Assignment Variable $\mathbf{a}^{0}(k)$. It is identical to the assignment in the standard MHT (Reid, 1979), thus

$$
P\left\{\mathbf{a}^{0}(k) \mid \mathbf{A}^{k-1, s}, Z^{k-1}\right\}=\frac{N_{D}}{m(k) !} \mu_{n+f} P_{D}^{N_{D}}\left(1-P_{D}\right)^{N_{T G T}-N_{D}}
$$

where $P_{D}$ is the detection probability, $N_{T G T}$ is the number of existing targets, $\mu_{n+f}$ is the new measurement or clutter density, which is assumed to be uniform in the whole surveillance region.

2. Doppler order assignment of each measurement $\mathbf{a}^{0}(k)$ According to the previous uniform assumption (4.17), it is a constant. Therefore, simplifying and combining constants into $c$ results in

$$
\begin{aligned}
P\left\{\mathbf{A}^{k, l} \mid Z^{k}\right\}= & \frac{N_{D} !}{c \cdot m(k) !} \mu_{n+f} P_{D}^{N_{D}}\left(1-P_{D}\right)^{N_{T G T}-N_{D}} V^{-N_{N F}} \\
& \cdot \prod_{i \in \mathbf{a}_{T}(k)}^{m(k)} f_{t_{i}}\left(z_{i, k}^{U}\right)
\end{aligned}
$$




\subsection{JIPDA Algorithm}

In section 5.2, an optimal solution to the problem of multitarget tracking with Doppler ambiguity has been presented. However, in the standard form, notice that the computational load is still heavy for several reasons: 1) The total number of current associations events could rise exponentially with respect to the number of measurement receiving at current time. With respect to a fixed measurement-to-track association, various Doppler assignment for each measurement can be chosen. For example, $N_{0}$ measurement-to-track assignments are generated, but the total number of track-measurement-Doppler assignment could be up to $\left(2 N_{d}\right)^{m(k)} N_{0}$, where $m(k)$ is the number of current measurement and $N_{d}$ is the number of possible Doppler orders. With valid gating strategy, instead of all possible values, only the Doppler order falling in the valid gate can be chosen for each measurement, where the gate is according to prediction. However, the number of spring-out hypotheses is still large when the measurements are abundant. 2) Because the hypothesis is a combination of all history association events, the number of hypothesis grows exponentially over time. With strategies referred in section 4.2 , such as pruning and merging, the total number of hypotheses can be limited to make the MHT practical (Bar-Shalom and $\mathrm{Li}, 1995)$. In this paper, the hypotheses that survived after pruning, might lose the generality of the samples. For example, all the association are identical for the measurement-to-track association, varying only in the Doppler ambiguity association for some measurements. The loss of generality would degrade the tracking performance, e.g., tracking divergence or track breakage, which are not expected.

Notice that the joint events are defined by the assignment variable in equation (5.1) and (5.5), which yield the problem of track-measurement-Doppler order 3D 
assignment. As the same unifying strategy in (Bojilov et al., 2002), this 3D problem is broken down in two steps:

1. For measurement $z_{i, k}$, all possible candidates with different Doppler orders are unified into one merged measurement. Notice that this unifying process is a PDA method that calculate the weighted sum of state estimation updated with all possible measurements.

2. After that, associate the existing tracks with the merged measurement.

Observe that the original 3D association problem is divided into to $2 \mathrm{D}$ problem and a Doppler ambiguity for single target tracking. The purpose of the dividing strategy is to decrease the number of events chosen, without losing the weight of the chosen subset from all feasible assignments. Because each assignment event in step 2, is actually a merged result of a set of events in the original 3D problem, who share the same track-measurement association but differ from the Doppler ambiguity for some measurements. For the first level ambiguity merging, the PDA, which is presented in detailed in section 4.3 is used. The second level association, the JIPDA algorithm is chosen here.

The joint probabilistic data association (JPDA) (Musicki and Evans, 2004) filter handles the possible presence of multiple targets in a joint JPDAD (Bar-Shalom and Li, 1995) manner. The JPDAF algorithm allows for the possibility that a measurement may have originated from one of a number of candidate tracks or clusters. In each scan, all possible joint measurement-to-track assignments are generated, which are called joint events, and calculated the posterior probability of the each joint events. Based on the probability for each event, the data association coefficient can be calculated for each track, with which the track estimates would be updated. Compared 
with MHT, Instead of the all history assignment, only the assignment for the current scan is considered in JPDA. There will not be a problem of storing and updating a set of hypotheses parallel, which is the essential drawback of MHT. In addition, JPDA is a track oriented method, leading to conveniency and consistency in target management.

JIPDA is the same as JPDA frame but brings in the concept of target existence in order to achieve the track maintenance. Target existence is modeled as a Markov process, with two propagation models. Markov Chain One is considered here, detailed as follows.

Markov Chain one, which is first used in (Bar-Shalom et al., 1989), has two states: the target exists with a detection probability $P_{D}$, which is defined as event $\chi_{k}^{t}$ at time $k$ for track $t$, or the target dose not exist

The transition matrix between these two states is given as $\left[\begin{array}{ll}p_{11} & p_{12} \\ p_{21} & p_{22}\end{array}\right]$ and it holds that

$$
P\left\{\chi_{k}^{t}\right\}=p_{11} P\left\{\chi_{k-1}^{t}\right\}+p_{21}\left(1-P\left\{\chi_{k-1}^{t}\right\}\right)
$$

Usually, it is assumed that a dead track would never revive, so that $p_{21}=0$.

In each scan, tracks are partitioned into clusters. For Markov Chain One model, the priori estimated number of clutter measurements $\hat{m}_{k}$ in the cluster is

$$
\hat{m}_{k}=\sum_{i=1}^{m_{k}}\left(\prod_{t=1}^{T}\left(1-\frac{P_{D}^{t} P_{W}^{t} P\left\{\chi_{k}^{t} \mid Z^{k-1}\right\}}{m_{k}^{t}}\right)^{\mu(k, t, i)}\right)
$$

where $\mu(k, t, i)$ is one if measurement $\mathrm{i}$ is in the windows of track $t$ at scan $k$ and zero otherwise.

Let $\chi_{i}$ and $X$ denote the joint event $i$ and the number of joint event. Let $T_{0}^{i}$ 
and $T_{1}^{1}$ denote the set of tracks allocated no measurement and one measurement, respectively, in the joint event $\chi_{i}$. For standard JIPDA with Markov Chain One, nonparametric version, the posterior probability of the joint event $\chi_{i}$ becomes

$$
P\left\{\chi_{i} \mid Z^{k}\right\}=C^{-1} \prod_{t \in T_{0}^{i}}\left(1-P_{D}^{t} P_{W}^{t} P\left\{\chi_{k}^{t} \mid Z^{k-1}\right\}\right) \prod_{t \in T_{1}^{i}}\left(P_{D}^{t} P_{W}^{t} P\left\{\chi_{k}^{t} \mid Z^{k-1}\right\} \frac{f_{t}\left(z_{m(i, t), k}\right) V_{k}}{\hat{m}_{k}}\right)
$$

where $P_{W}^{t}$ is the probability that measurements falling into the valid gate of track $t, m(i, t)$ denotes the measurement, which is allocated to track $\mathrm{t}$ under joint event $\mathrm{i}$, and $f_{t}\left(z_{m(i, t), k}\right)$ is the likelihood of this merged measurement. Thus

$$
f_{t}\left(z_{m(i, t), k}\right)=P\left\{z_{m(i, t), k} \mid Z^{k}\right\}
$$

The normalization constant $\mathrm{C}$ is calculated by

$$
\sum_{j=1}^{X} P\left\{\chi_{j} \mid Z^{k}\right\}=1
$$

The a posteriori probability of an individual track event is obtained by summing the a posteriori probabilities of all joint events containing the track event. Denoted by $\Xi(t, i)$, the set of joint events in which track t has been allocated measurement i ( 0 denoting no measurement). The following posteriori probabilities in the JIPDA can be calculated

1. no measurement originating from the track $t$

$$
P\left\{\chi_{k, 0}^{t} \mid Z^{k}\right\}=\sum_{e \in \Xi(t, 0)} P\left\{\chi_{e} \mid Z^{k}\right\}
$$


2. the event of target $t$ exists with no measurement originating from the target

$$
P\left\{\chi_{k}^{t}, \chi_{k, 0}^{t} \mid Z^{k}\right\}=\frac{\left(1-P_{D}^{t} P_{W}^{t}\right) P\left\{\chi_{k}^{t} \mid Z^{k-1}\right\}}{1-P_{D}^{t} P_{W}^{t} P\left\{\chi_{k}^{t} \mid Z^{k-1}\right\}} P\left\{\chi_{k, 0}^{t} \mid Z^{k}\right\}
$$

3. the event of target $t$ exists with measurement $i$ originating from the target

$$
P\left\{\chi_{k}^{t}, \chi_{k, i}^{t} \mid Z^{k}\right\}=\sum_{e \in \Xi(t, i)} P\left\{\chi_{e} \mid Z^{k}\right\}
$$

4. the existence probability of track $t$

$$
P\left\{\chi_{k}^{t} \mid Z^{k}\right\}=\left\{\chi_{k}^{t}, \chi_{k, 0}^{t} \mid Z^{k}\right\}+\sum_{i} P\left\{\chi_{k}^{t}, \chi_{k, i}^{t} \mid Z^{k}\right\}
$$

Then the $\beta$ data association probability for track $t$ are

$$
\begin{aligned}
& \beta_{0}^{t}=\frac{P\left\{\chi_{k}^{t}, \chi_{k, 0}^{t} \mid Z^{k}\right\}}{P\left\{\chi_{k}^{t} \mid Z^{k}\right\}} \\
& \beta_{i}^{t}=\frac{P\left\{\chi_{k}^{t}, \chi_{k, 0}^{t} \mid Z^{k}\right\}}{P\left\{\chi_{k}^{t} \mid Z^{k}\right\}}
\end{aligned}
$$

Notice that in the standard JIPDA, event $\chi_{i}$ only determines the measurement-totrack association, with the Doppler order for each assigned measurement still unfixed. When the assignment is expended from $2 \mathrm{D}$ to $3 \mathrm{D}$, it can be seen that there is a set of events who share the same track to measurement association $\chi_{i}$. For example, with respect to a fixed measurement-to-track association e.g., event i, each Doppler assignment is an valid candidate., The same method has been applied to the Interacting Multiple Model (IMM) JPDA in (Bojilov et al., 2003) (Bojilov et al., 2002). 
Therefore

$$
\begin{aligned}
P\left\{\chi_{i} \mid Z^{k}\right\} & =\sum_{n} P\left\{\chi_{i}, a(k, m, t, n) \mid Z^{k}\right\} \\
& =\sum_{n} P\left\{\chi_{i} \mid a(k, m, t, n), Z^{k}\right\} P\left\{a(k, m, t, n) \mid Z^{k}\right\} \\
& =\sum_{n} P\left\{\chi_{i} \mid a(k, m, t, n), Z^{k}\right\} P\{a(k, m, t, n)\}
\end{aligned}
$$

Observe that with determined $a(k, m, t, n)$, the likelihood term $f$ in equation(5.17) is fixed. The prior distribution of Doppler order for measurement $m$ is uniform. According to the analysis, with respect to track $t_{0}$ (assume it is associated with measurement $m_{0}$ in event i), equation(5.17) can be written as

$$
\begin{aligned}
P\left\{\chi_{i} \mid Z^{k}\right\}= & \sum_{n} P\left\{\chi_{i} \mid a\left(k, m, t_{0}, n\right), Z^{k}\right\} P\left\{a\left(k, m, t_{0}, n\right)\right\} \\
= & \sum_{n} C^{-1} \prod_{t \in T_{0}^{i}}\left(1-P_{D}^{t} P_{W}^{t} P\left\{\chi_{k}^{t} \mid Z^{k-1}\right\}\right) \\
& \cdot \prod_{t \in T_{1}^{i}, t \neq t_{0}}\left(P_{D}^{t} P_{W}^{t} P\left\{\chi_{k}^{t} \mid Z^{k-1}\right\} \frac{f_{t}\left(z_{m(i, t), k}\right) V_{k}}{\hat{m}_{k}}\right) \cdot \frac{f_{t_{0}}\left(z_{a\left(k, t_{0}, m_{0}, n\right.}\right) V_{k}}{\hat{m}_{k}} N^{-1} \\
= & C^{-1} \prod_{t \in T_{0}^{i}}\left(1-P_{D}^{t} P_{W}^{t} P\left\{\chi_{k}^{t} \mid Z^{k-1}\right\}\right) \cdot \prod_{t \in T_{1}^{i}, t \neq t_{0}}\left(P_{D}^{t} P_{W}^{t} P\left\{\chi_{k}^{t} \mid Z^{k-1}\right\}\right. \\
& \left.\left.\frac{f_{t}\left(z_{m(i, t), k}\right) V_{k}}{\hat{m}_{k}}\right)\left(\sum_{n} N^{-1} f_{t_{0}}\left(z_{a\left(k, t_{0}, m_{0}, n\right.}\right)\right) \frac{V_{k}}{\hat{m}_{k}}\right)
\end{aligned}
$$

Defined the merged likelihood as

$$
\bar{f}_{t}\left(z_{m(i, t), k}\right)=\sum_{n} N^{-1} f_{t_{0}}\left(z_{a\left(k, t_{0}, m_{0}, n\right.}\right)
$$

and replace $f_{t}\left(z_{m(i, t), k}\right)$ with the merged likelihood for each associated measurement 
to get

$$
P\left\{\chi_{i} \mid Z^{k}\right\}=C^{-1} \prod_{t \in T_{0}^{i}}\left(1-P_{D}^{t} P_{W}^{t} P\left\{\chi_{k}^{t} \mid Z^{k-1}\right\}\right) \prod_{t \in T_{1}^{i}}\left(P_{D}^{t} P_{W}^{t} P\left\{\chi_{k}^{t} \mid Z^{k-1}\right\} \frac{\bar{f}_{t}\left(z_{m(i, t), k}\right) V_{k}}{\hat{m}_{k}}\right)
$$

Therefore, all possible unambiguous measurements, based on the same true observation, have a contribution to the probability of the measurement-to-track association event, weighted by the likelihood. On the other hand, each event $\chi_{i}$ covers all possible combinations of the Doppler order for each measurement, which, yields to the rapid reduction of the total number of events. 


\section{Chapter 6}

\section{SIMULATION AND \\ PERFORMANCE EVALUATION}

\subsection{Single Target}

\subsubsection{Simulation Scenario}

A single target is simulated by model (3.5) in a clean environment with the following parameters. For the target, initial state is $x_{0}=[1000,1000,10,10]^{\prime}$ and process noise is set to $\sigma_{x}=\sigma_{y}=0.5 \mathrm{~m} / \mathrm{s}^{2}$. For the radar system, the first blind velocity for the simulated measurement is $10 \mathrm{~m} / \mathrm{s}$, which is a reasonable value for the Doppler radar system. The standard deviation of measurement noise is $\sigma_{r}=100 \mathrm{~m}, \sigma_{\theta}=$ $0.05 \mathrm{rad}, \sigma_{\dot{r}}=\sigma_{\Delta \dot{r}}=1 \mathrm{~m} / \mathrm{s}$.

Notice that the error of the position measurement are amplified in order to test the contribution of the Doppler measurement to the performance. The target will 
be tracked with three different measurements : 1) Position only $[r, \theta], 2)$ Ambiguous Doppler measurement $[r, \theta, \Delta \dot{r}]$, 3) Unambiguous Doppler measurement $[r, \theta, \dot{r}]$ respectively. Their track performance, including position and velocity RMSE, will be compared with each other, where the position and velocity RMSE $e_{p}$ and $e_{v}$ are defined as

$$
\begin{aligned}
& e_{p}(k)=E\left[\sqrt{\tilde{x}_{k}^{2}+\tilde{y}_{k}^{2}}\right] \\
& e_{v}(k)=E\left[\sqrt{\tilde{\dot{x}}_{k}^{2}+\tilde{\dot{y}}_{k}^{2}}\right]
\end{aligned}
$$

where $\left[\tilde{x}_{k}, \tilde{y}_{k}, \tilde{\dot{x}}_{k}, \tilde{y}_{k}\right]$ is the error between the true state and the estimation at time $k$.

Meanwhile, to solve the Doppler ambiguity for the second kind of measurements (with Doppler ambiguity), the aforementioned three methods are implemented respectively and solved with three methods: UKF modification, MHT and PDA. For MHT and PDA algorithms, UKF tracker is used only for the nonlinear tracking.

\subsubsection{Performance Evaluation}

In most cases, with the points shifting and closest innovation, the UKF can solve the Doppler ambiguity and can achieve the same track performance as that with unambiguous one, as shown in Fig 6.1(a), where the tracking results with or without Doppler ambiguity overlap with each other.

However in some instances as seen before, such as when $\max \left(\mathcal{Z}_{i, t \mid t-1}^{U}\right)-\left(\mathcal{Z}_{0, t \mid t-1}^{U}\right) \leq$ $v_{f} / 2$, or $\left|\nu^{U}\right|>\min _{\Delta n}\left|\nu^{A}-\Delta n_{d} v_{f}\right|$, UKF would implement with a false estimation of mean and covariance or an incorrect innovation, which might lead to a divergence estimation, as shown in Fig. 6.1(b). The reason is that under an inappropriate 


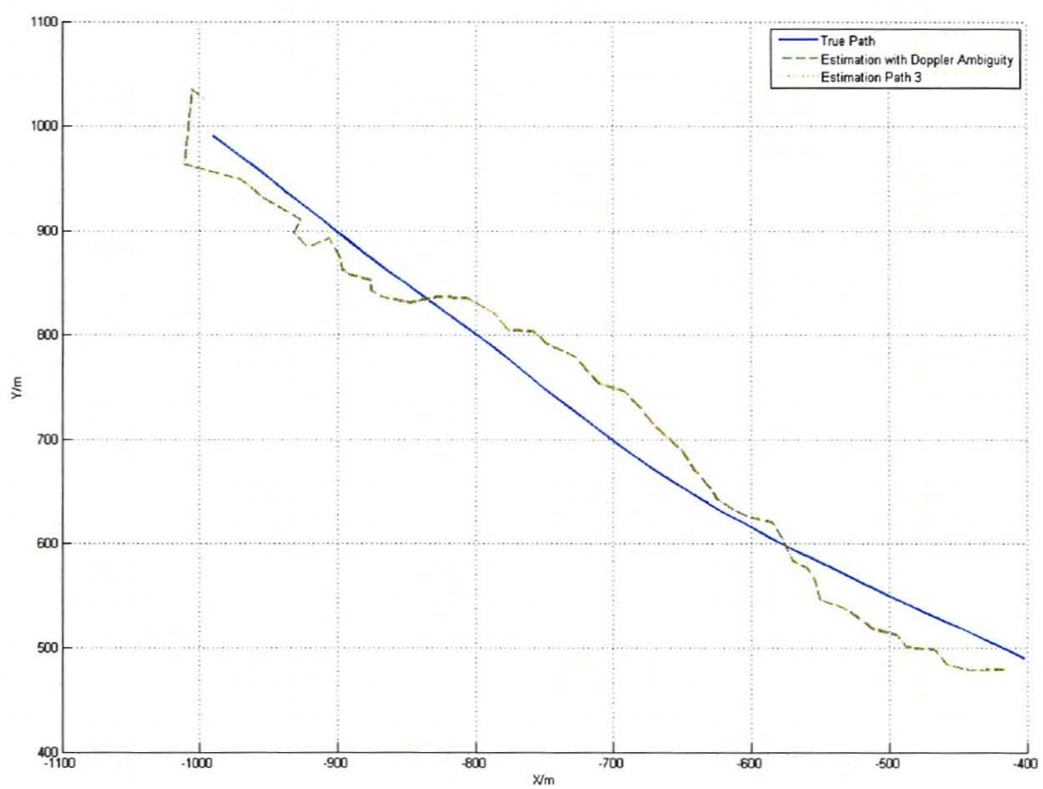

(a) Good case

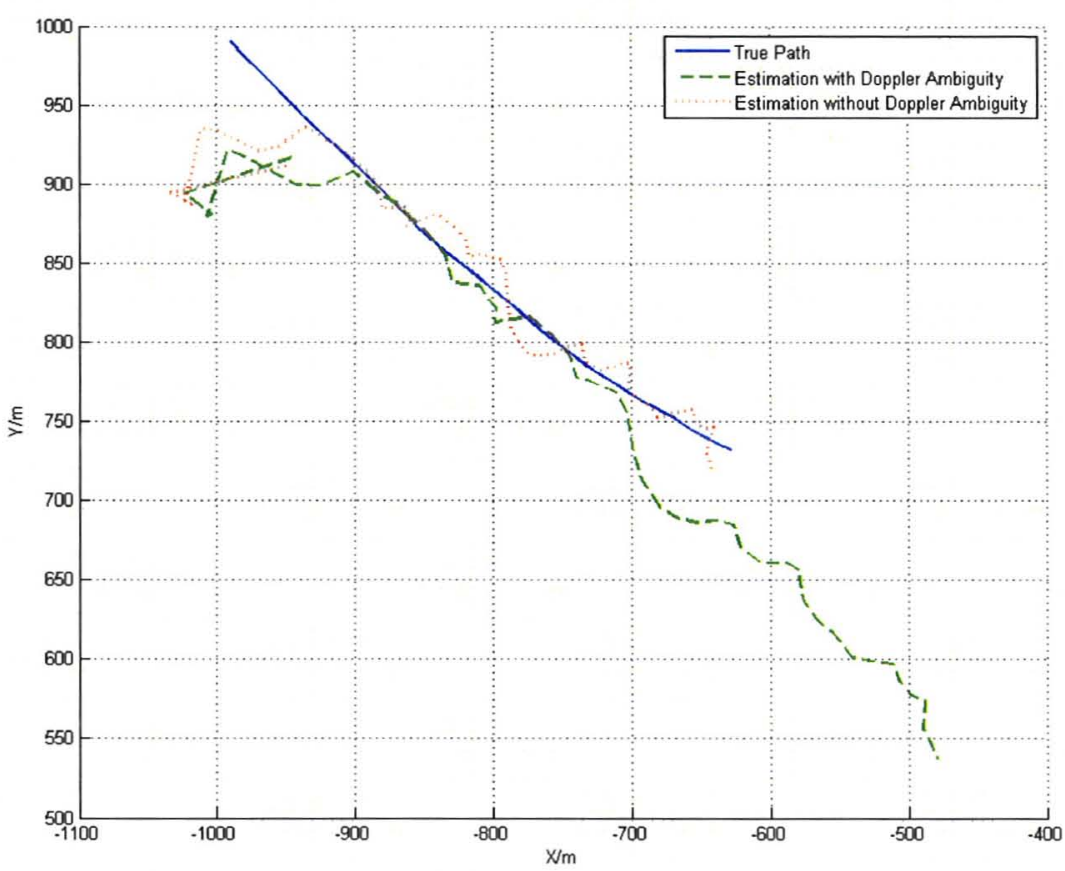

(b) Divergence case

Figure 6.1: UKF ${ }_{46}$ tracking results 
Table 6.1: UKF for Doppler ambiguity

\begin{tabular}{|c|c|c|}
\hline & Unambiguous & Ambiguous \\
\hline Doppler measurement(m/s) & 12.65 & $2.65, v_{f}=10 \mathrm{~m} / \mathrm{s}$ \\
\hline Sigma Points & $19.08,22.25,21.86,19.16,18.71$, & $9.08, \underline{2.25}, \underline{1.86,9.16,8.71,}$ \\
& $15.90,16.29,18.65,19.28$ & $5.90,6.29,8.65,9.28$ \\
\hline Modified Sigma points & N/A & $9.08, \underline{12.25}, \underline{11.86}, 9.16,8.71$, \\
& & $5.90,6.29,8.65,9.28$ \\
\hline Mean $\bar{z}$ & 19.02 & 9.02 \\
\hline Covariance $P_{z z}$ & 4.29 & 4.29 \\
\hline Innovation & $\nu=-6,37$ & $\nu^{*}=3.63$ \\
\hline
\end{tabular}

initialization or high maneuvering condition, the accuracy assumption is not valid at all. Table 6.1 shows how divergence happens with simulation data.

In the example table 6.1, for both ambiguous or unambiguous Doppler measurements, nine sigma points of the measurement prediction are generated first by UKF algorithm. Notice that only the Doppler components of these sigma points are listed. And it has been seen that these sigma points with ambiguity in the second column, have been shifted by $-v_{f}$ or $-2 v_{f}$ because of the mod operation. By using the UKF modification, two underlined points $\underline{2.25}, \underline{1.86}$ are shifted to $\underline{12.25}, \underline{11.86}$ because $\left|z_{i}-z_{0}\right|>v_{f} / 2$, which holds the covariance of the measurement prediction compared to the data in the first column. The innovation calculated from the data in the first column is $\nu=-6,37$. However, based on the shortest distance between the prediction and measurement, the optimal innovation for ambiguous Doppler measurement obtained from the sigma points in the second column is $\nu^{*}=3.63$, shifted by $v_{f}$. This incorrect innovation leads to an estimation on the opposite way to the true target movement, which is, in another word, divergency. This divergency problem is a cause for concern especially when the Doppler measurement is accurate $\left(\sigma_{\dot{r}}\right.$ is small). In other words, the Doppler component of the innovation influence the final 
estimation to a great degree. The reasons for the incorrect choice innovation, or poor prediction of the Doppler measurement, can be summarized into two factors: Poor estimation or initialization and high maneuvering.

However, the divergence is a low probability event, e.g. 322 out of 10,000 times run as shown in table 6.2. Beside divergence events, the proposed UKF method can solve the Doppler ambiguity correctly and achieve a better performance compared to the estimation with position only measurements. In Fig. 6.2, it can be seen that by the proposed algorithm, the position and velocity RMSE of the ambiguous Doppler case almost overlap with that of the unambiguous case.

One the other hand, the MHT is able to fully solve the ambiguity problem. However, with limitation of the total number of the hypotheses, which is expected to increase exponentially with time, the prune threshold $P_{t h}$ is set to delete the hypotheses with a smaller probability than the pruning threshold. However, the correct hypothesis, might have a low probability at the beginning, especially in a poor initialization system, such as one point initialization. Thus, even thought the correct hypothesis could dominate over others at last, it had been deleted with this pruning strategy. The best way to keep the correct hypothesis is to set a lower pruning threshold, which, on the other hand, brings to the problem of larger computation load for numbers of existing hypotheses to be processed. Simulations are processed under three different pruning threshold probabilities, $P_{\text {th }}=0.05,0.001,0.001$, and 0.0001 . Like previous analysis, the rate of divergence has been counted in each condition (including using UKF solving ambiguity), summarized in table 6.2.

From the table, observe that with the large value of prune threshold probability, e.g., $P_{t h}=0.05$, the divergence still exists, but the rate at which it happens has 
Table 6.2: The Divergence Rate

\begin{tabular}{|l|c|}
\hline Method \& threshold & Divergence rate \\
\hline UKF & $3.22 \%$ \\
\hline MHT, $p_{t h}=0.05$ & $0.93 \%$ \\
\hline MHT, $p_{t h}=0.001$ & $0.10 \%$ \\
\hline MHT, $p_{t h}=0.0005$ & $0.06 \%$ \\
\hline MHT, $p_{t h}=0.0001$ & 0 \\
\hline PDA, & $1.1 \%$ \\
\hline
\end{tabular}

been decreased by almost $70 \%$ compared with the rate in first row when using UKF. With choosing appropriate value of this threshold, e.g., $P t_{3}=0.0001$, there is no divergence at all. In other words, the Doppler ambiguity has been totally solved in this condition.

After eliminating the "bad" runs, the position and velocity RMSE of the tracker are shown in Fig. 6.2. Observe that by the proposed MHT algorithm, the position and velocity RMSE of the ambiguous Doppler case overlap with that of the unambiguous case. Therefore, the Doppler ambiguity has been solved, and with the help of the ambiguous Doppler measurement, the tracking performance has been increased by more than $40 \%$.

Compared to MHT, PDA would provide a suboptimal solution of the Doppler ambiguity but in less time. The RMSE and the Divergence rate for the PDA method are shown in Fig. 6.2 and table 6.2, respectively. 


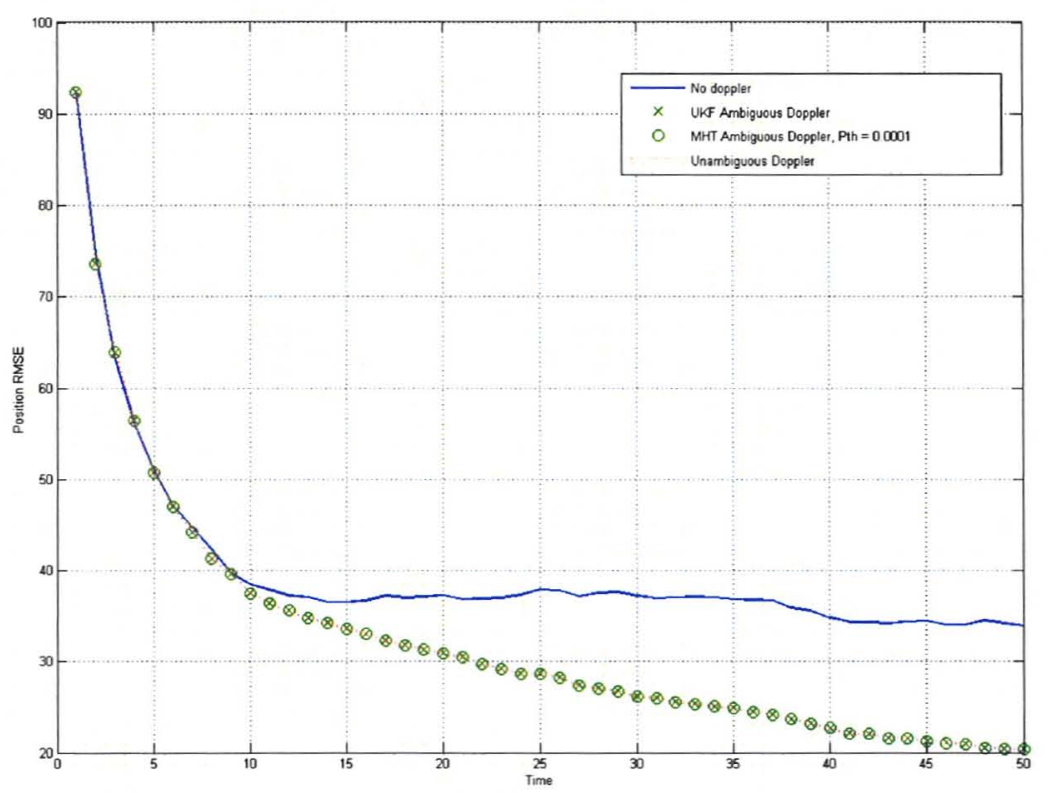

(a) Position RMSE

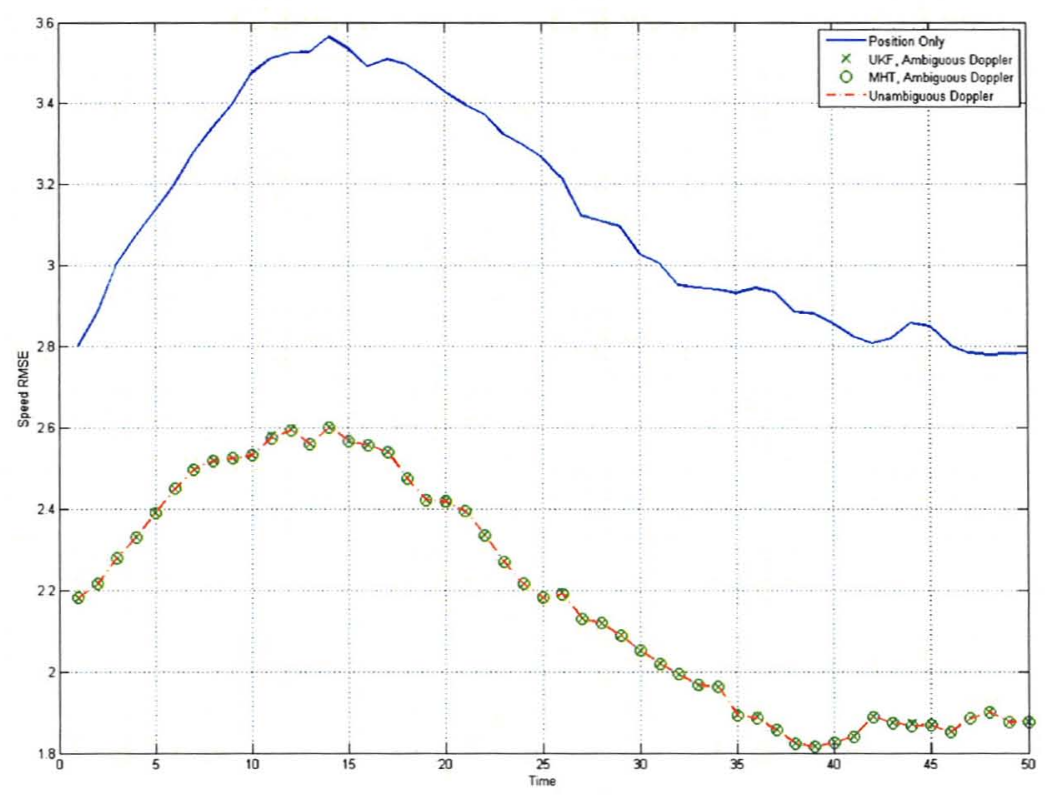

(b) Velocity RMSE

Figure 6.2: RMSE for UKF and MHT 


\subsection{Multitarget}

\subsubsection{Simulation Scenario}

The multitarget tracking problem is simulated. Four targets, propagating with model (3.5) with white acceleration noise, are simulated in the surveillance region where the starting time and initial state for each target are listed in table 6.3 and the trajectory of each target is shown in Fig 6.3. Notice that target 1 and target 4 are overlapped around time $k=22$, but with different speed. The process noise is set to $\sigma_{x}=\sigma_{y}=0.05 \mathrm{~m} / \mathrm{s}^{2}$. For the sensor, first blind velocity is set to be $v_{f}=10 \mathrm{~m} / \mathrm{s}$ and the measurement error are $\sigma_{r}=5 \mathrm{~m}, \sigma_{\theta}=0.01 \mathrm{rad}, \sigma_{\Delta \dot{r}}=0.7 \mathrm{~m} / \mathrm{s}$. On the other hand, the false alarms are generated around the whole surveillance region, with a high clutter density in the center region as indicated by the black points in Fig 6.3. In a real scenario, the distribution of the Doppler measurement of the clutter points depends on the source of the clutter, such as stable objects, trees or tides. Therefore the Doppler measurements for clutters should be located in the low speed region. However, without losing generality in this simulation, it is assumed that the Doppler measurement for these clutter points have a uniform distribution over the region $\left(0, v_{f}\right)$.

The target will be tracked with three different measurements : 1) Position only $[r, \theta], 2)$ Ambiguous Doppler measurement $[r, \theta, \Delta \dot{r}]$ and 3) Unambiguous Doppler measurement $[r, \theta, \dot{r}]$ respectively. Both MHT and JIPDA trackers are implemented with the above three kinds of measurements. 
Table 6.3: The Initial State of Simulated targets

\begin{tabular}{|l|c|c|c|c|c|}
\hline Target No. & Starting time $(\mathrm{s})$ & Initial $x(\mathrm{~m})$ & Initial $y(\mathrm{~m})$ & Initial $\dot{x}(\mathrm{~m} / \mathrm{s})$ & Initial $\dot{y}(\mathrm{~m} / \mathrm{s})$ \\
\hline target 1 & 10 & 200 & 200 & 10 & 10 \\
\hline target 2 & 10 & 200 & 700 & 10 & -10 \\
\hline target 3 & 0 & 100 & 400 & 10 & 2 \\
\hline target 4 & 0 & 200 & 200 & 5 & 5 \\
\hline
\end{tabular}

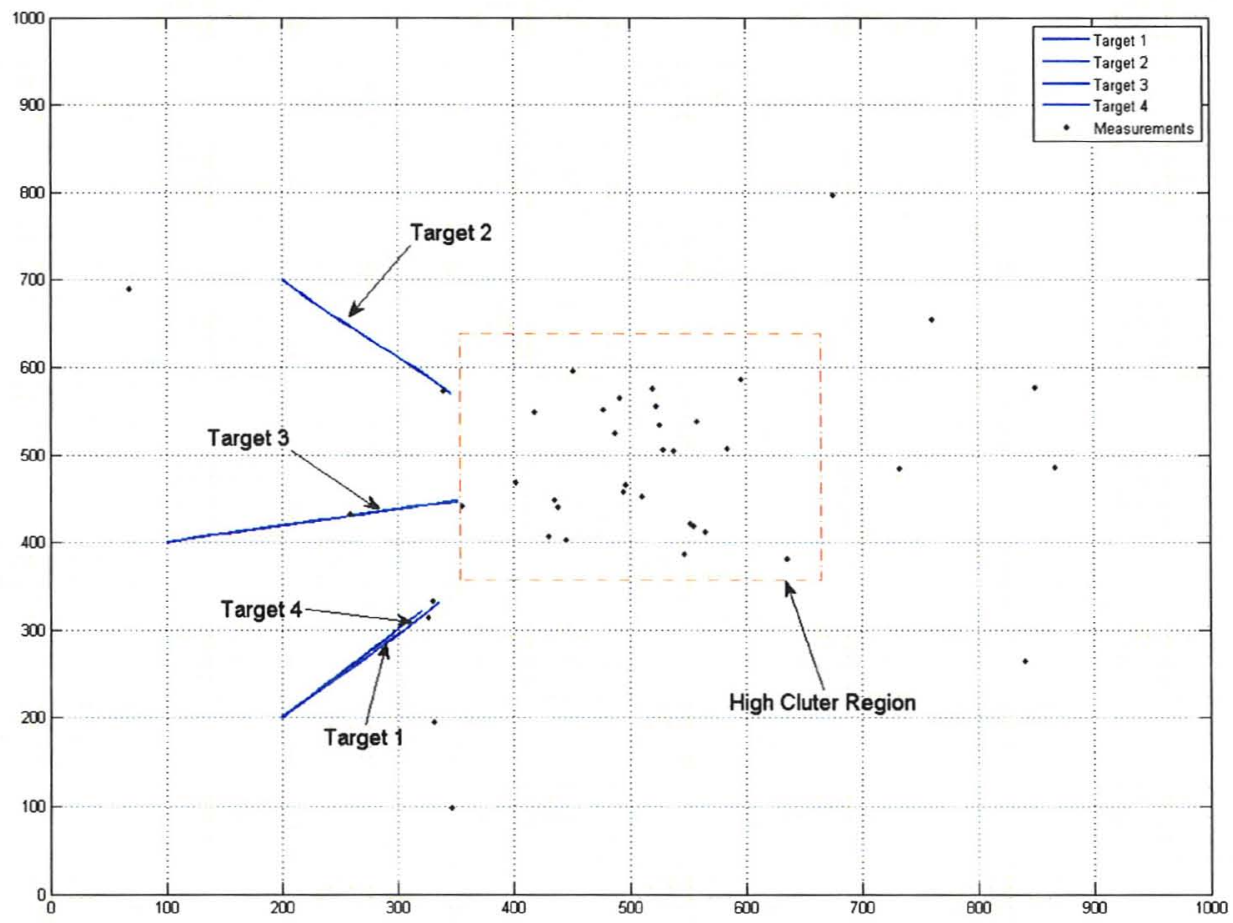

Figure 6.3: Target Trajectory and Measurements 


\subsubsection{Performance Evaluation}

The following figure gives one time simulation at a certain scan, Fig. 6.4. The lines indicate the tracking results with three different kind of measurements as well as the true trajectory of these targets. The performance of three tracking results can be

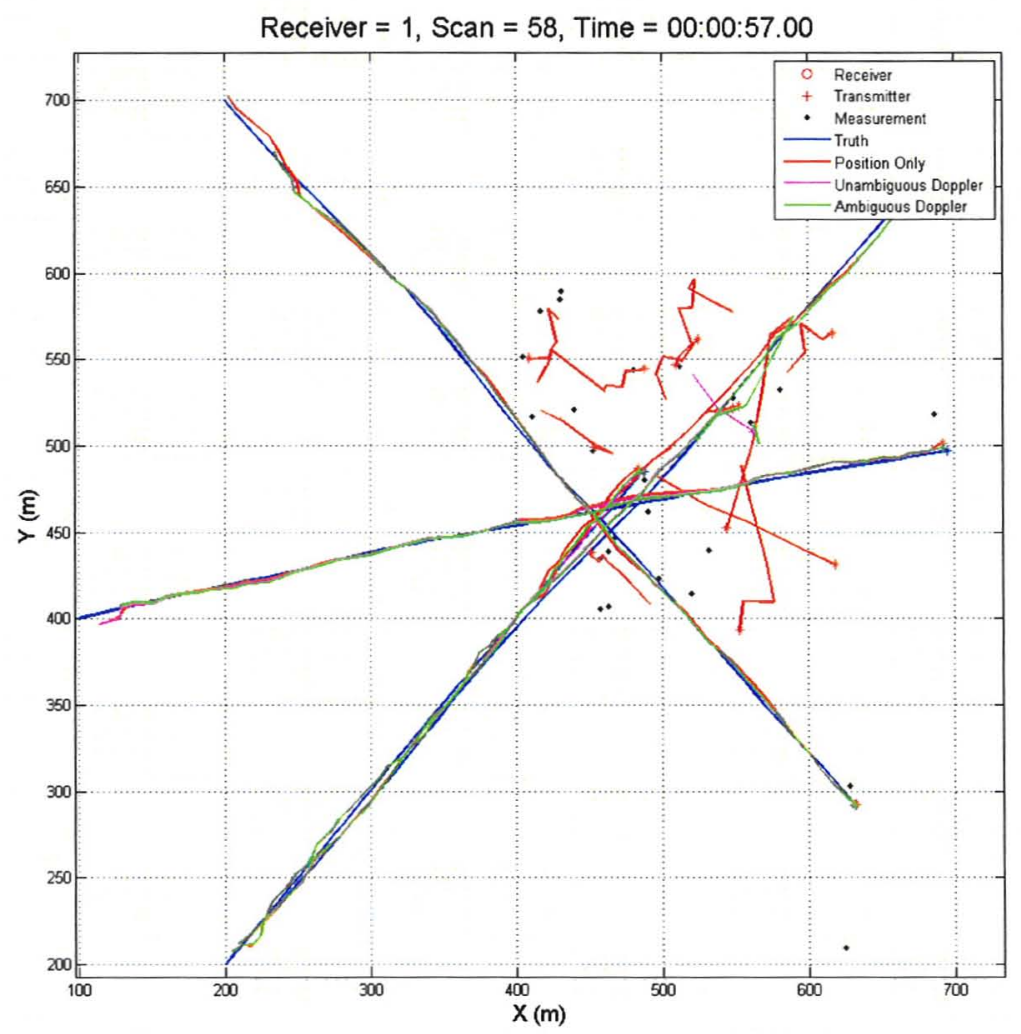

Figure 6.4: Tracking Results

compared in the following two aspects: RMSE and number of false tracks.

Without loosing generality, all tracker's results for target 2 are compared. The position RMSE of MHT and JIPDA trackers are shown in Fig.6.5(a) and Fig.6.5(b), respectively. The solid line indicates the RMSE curve for position only measurement and the dashed line indicates that for Doppler measurement. Observe that the RMSE 


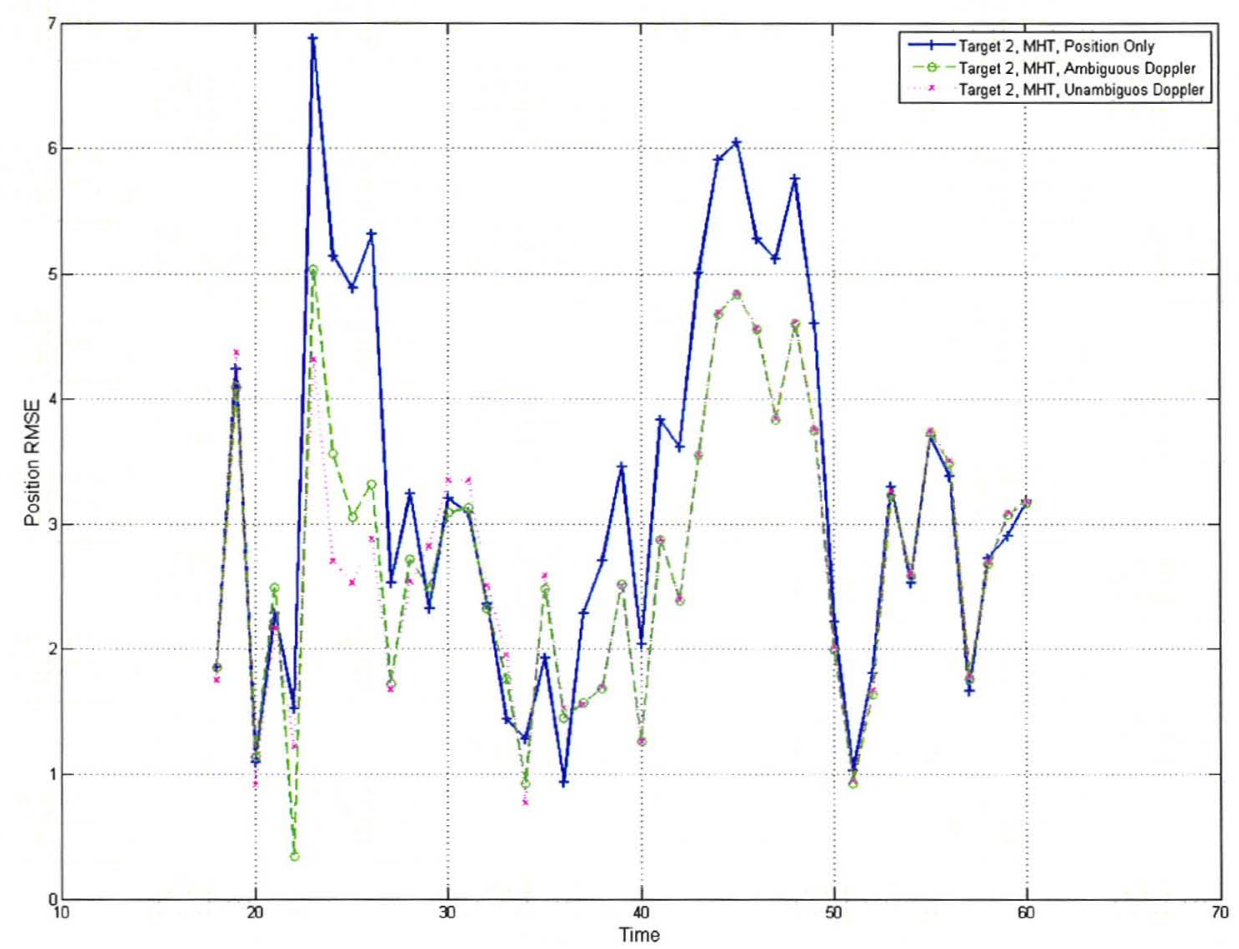

(a) Position RMSE for MHT, Target 2

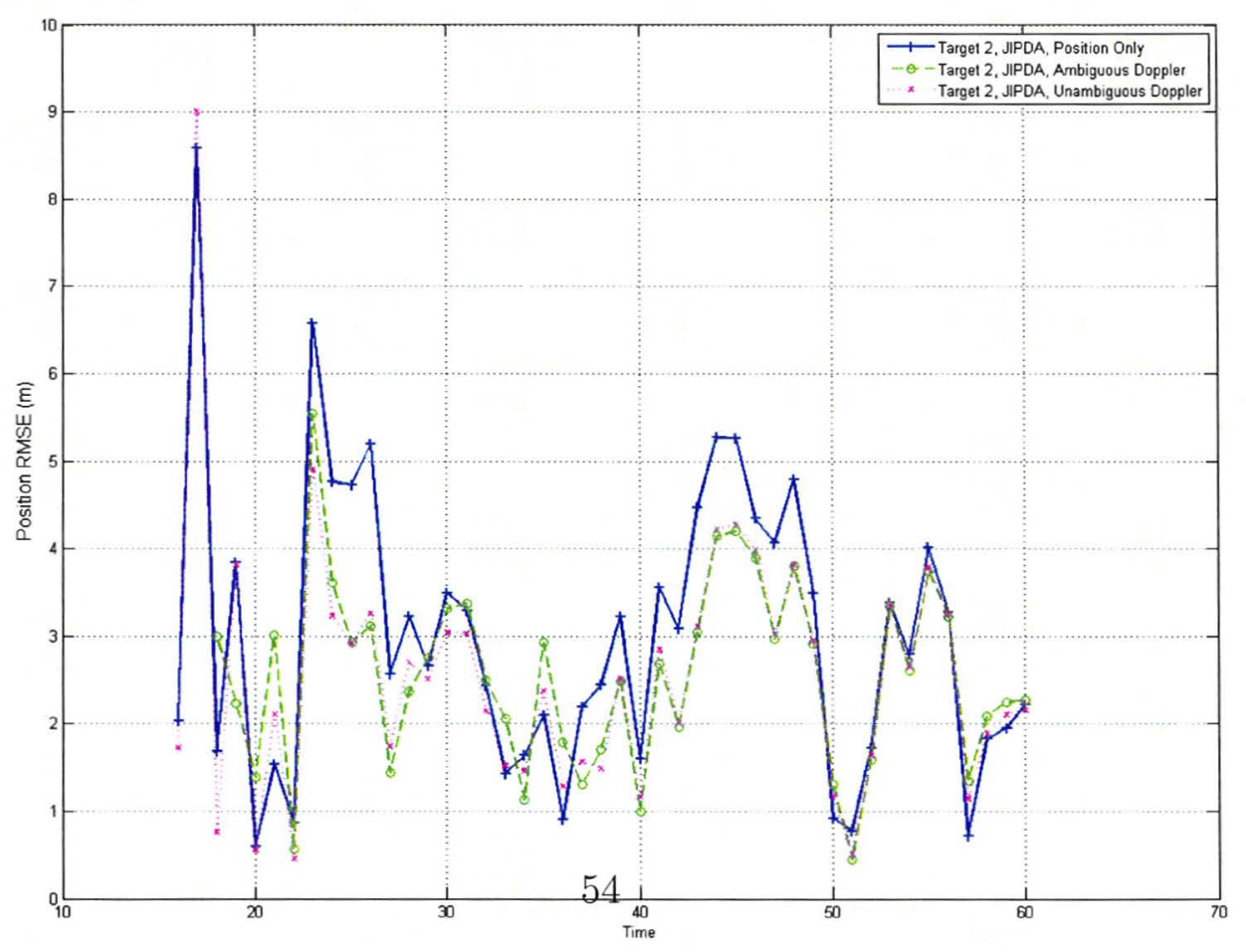


has been reduced with the introduction of the Doppler measurement, both for MHT and JIPDA tracker. Furthermore, for MHT in Fig.6.5(a), although there is a noticeable difference between the RMSE of ambiguous Doppler (dashed line with circle) and unambiguous one (dashed line with cross) at the first 30 time scans, these two curves overlap each other after time $k=35$, clearly indicating that the Doppler ambiguity is completely solved after then. On the other hand, in JIPDA, the RMSE of ambiguous Doppler approach to the ambiguous Doppler curve faster but an acceptable difference between two lines exist at all time.

Secondly, observe that in the surveillance region, especially in the high clutter region, a large number of false tracks, which do not exist in real, have been generated with the position measurement. This is expected when two clutter measurement points (position only) in consecutive scans have short distance between each other and a false track is likely to be generated with these two points. However, with the Doppler measurement, even though such point pair has a short distance in position space, it is possible to make a more strict judgement whether or not to associate these two points by using the distance in Doppler space. The false track rate is defined as the average number of false tracks per time scan, which is

$$
R_{f}=\frac{1}{T_{\text {sim }}} \sum_{k=0}^{T_{s i m}} N_{f}(k)
$$

where $T_{\text {sim }}$ is the total simulation time and $N_{f}(k)$ is the number of false tracks at time $k$.

The false track rates of three track results are compared in the first row in table 6.4. Observe that with using the Doppler measurement, the false alarm rate has been decreased from 2.046 to 0.306 for JIPDA tracker and from 5.048 to 3.177. The 
differences between the JIPDA and MHT is due to different tracker initialization and management strategy. Therefore, it is not reasonable to make a judgement that JIPDA is better than MHT from this point of view.

Table 6.4: False Alarm rate

\begin{tabular}{|l|c|c|c|}
\hline & Position Only & Ambiguous Doppler & Unambiguous Doppler \\
\hline MHT & 5.048 & 3.177 & 0.371 \\
\hline JIPDA & 2.046 & 0.306 & 0.065 \\
\hline
\end{tabular}

With the help of the ambiguous Doppler measurement, the false alarm rate has been decreased by up to $85 \%$. However, compared the optimal case without ambiguity, a better ambiguity solving method for the multitarget tracking need to be further studied. 


\section{Chapter 7}

\section{CONCLUSION}

In this paper a detailed study of the Doppler measurement in PRF radar system was presented, which provides extra information of the range rate, but usually comes with the ambiguity problem.

To utilize this range rate information, several methods were proposed to solve this Doppler ambiguity in single target tracking problem. First, based on UKF, the sigma points were modified to hold the statistic property of the variable (state estimation) in a nonlinear process. With such modification, a better performance of tracking estimation can be achieved with modified UKF. To solve the Doppler ambiguity with the history measurements, methods based on MHT and PDA, both of which are used to solve the measurement-to-track association, were used to resolve the problems considered. This paper also extended the Doppler ambiguity problem into the multitarget tracking in cluttered environment. The analytical expression of solving the problem via standard MHT was demonstrated here. However, to overcome the disadvantage of the track-measurement-Doppler 3D association, the problem was 
decomposed into two 2D data association: track-measurement and measurementDoppler and deduce a suboptimal solution. JIPDA tracker was applied to implement this suboptimal algorithm for the purpose of higher efficient.

Finally, based on the simulation and real Doppler radar data, it has been proven that 1) For single target tracking, the Doppler Ambiguity can be fully solve by propose algorithm (MHT / PDA). Although Divergence happens, the RMSE of the tracking estimation is improved with Doppler measurement and the divergence rates are compared against different methods. 2) For multitarget tracking, the false alarm rate in the high clutter region is rapidly decreased with the use Doppler measurement. 


\section{Bibliography}

Alabaster, C., Hughes, E., and Matthew, J. (2003). Medium PRF radar PRF selection using evolutionary algorithms. Aerospace and Electronic Systems, IEEE Transactions on, 39(3), $990-1001$.

Bar-Shalom, Y. (2000). Negative Correlation and Optimal Tracking with Doppler Measurements. In Proceedings of SPIE, volume 4048, page 583.

Bar-Shalom, Y. and Li, X. (1995). Multitarget-Multisensor Tracking: Principles and Techniques. Storrs, CT: University of Connecticut.

Bar-Shalom, Y. and Tse, E. (1975). Tracking in a cluttered environment with probabilistic data association. Automatica, 11, 451-460.

Bar-Shalom, Y., Chang, K., and Blom, H. (1989). Automatic track formation in clutter with a recursive algorithm. In Decision and Control, 1989., Proceedings of the 28th IEEE Conference on, pages 1402 -1408 vol.2.

Bar-Shalom, Y., Li, X., and Kirubarajan, T. (2001). Estimation with Applications to Tracking and Navigation. New York: Wiley-Interscience.

Blackman, S. S. (1986). Multiple-target Tracking with Radar Applications. Dedham, MA, Artech House, Inc. 
Bojilov, L., Alexiev, K., and Konstantinova, P. (2002). An algorithm unifying IMM and JPDA approaches. COMPTES RENDUS-ACADEMIE BULGARE DES SCIENCES, 55(12), 27-34.

Bojilov, L., Alexiev, K., and Konstantinova, P. (2003). An Accelerated IMM JPDA Algorithm for Tracking Multiple Manoeuvring Targets in Clutter. Numerical Methods and Applications, 2542/2003, 274-282.

Ferrari, A., Berenguer, C., and Alengrin, G. (1997). Doppler ambiguity resolution using multiple PRF. Aerospace and Electronic Systems, IEEE Transactions on, $33(3), 738-751$.

Hovanessian, S. (1976). An Algorithm for Calculation of Range in a Multiple PRF Radar. Aerospace and Electronic Systems, IEEE Transactions on, AES-12(2), 287 $-290$.

Hughes, E. and Lewis, M. (2009). Improved Detection and Ambiguity Resolution of Multiple Targets in MPRF Radar. Cranfield Defence and Security.

Jazwinski, A. (1970). Stochastic Processes and Filtering Theory. Academic Pr.

Julier, S. and Uhlmann, J. (2004). Unscented Filtering and Nonlinear Estimation. Proceedings of the IEEE, 92(3), 401-422.

Koch, W. (2002). Effect of Doppler Ambiguities on GMTI Tracking. In RADAR 2002, pages 153-157.

Musicki, D. and Evans, R. (2004). Joint Integrated Probabilistic Data Association: JIPDA. Aerospace and Electronic Systems, IEEE Transactions on, 40(3), 10931099. 
Niu, R., Willett, P., and Bar-Shalom, Y. (2002). Tracking Considerations in Selection of Radar Waveform for Range and Range-Rate Measurements. Aerospace and Electronic Systems, IEEE Transactions on, 38(2), 467-487.

Reid, D. (1979). An algorithm for tracking multiple targets. Automatic Control, IEEE Transactions on, 24(6), 843-854.

Roecker, J. (1994). A Class of Near Optimal JPDA Algorithms. Aerospace and Electronic Systems, IEEE Transactions on, 30(2), 504-510.

Smith, M. (2008). On Doppler measurements for tracking. In Radar, 2008 International Conference on, pages 513-518.

Tharmarasa, R., Kirubarajan, T., Hernandez, M., and Sinha, A. (2007). PCRLBbased multisensor array management for multitarget tracking. Aerospace and Electronic Systems, IEEE Transactions on, 43(2), 539-555.

Tobias, M. and Lanterman, A. (2005). Probability hypothesis density-based multitarget tracking with bistatic range and Doppler observations. Radar, Sonar and Navigation, IEE Proceedings, 152(3), 195-205.

Trunk, G. and Brockett, S. (1993). Range and velocity ambiguity resolution. In Radar Conference, Record of the 1993 IEEE National, pages 146-149.

Tugnait, J. K. (1981). Detection and estimation for abruptly changing systems. In Decision and Control including the Symposium on Adaptive Processes, 20th IEEE Conference on, volume 20, pages 1357-1362. 
Wan, E. and Van Der Merwe, R. (2000). The unscented Kalman filter for nonlinear estimation. In Adaptive Systems for Signal Processing, Communications, and Control Symposium 2000. AS-SPCC. The IEEE 2000, pages 153-158.

Wang, X., Musicki, D., and Ellem, R. (2007). Fast track confirmation for multi-target tracking with doppler measurements. In Intelligent Sensors, Sensor Networks and Information. ISSNIP 2007. 3rd International Conference on, pages 263-268.

Wang, X., Musicki, D., Ellem, R., and Fletcher, F. (2009). Efficient and Enhanced Multi-Target Tracking with Doppler Measurements. Aerospace and Electronic Systems, IEEE Transactions on, 45(4), 1400-1417.

Xia, X.-G. (1999). Doppler Ambiguity Resolution Using Optimal Multiple Pulse Repetition Frequencies. Aerospace and Electronic Systems, IEEE Transactions on, 35(1), 371-379.

Yeom, S.-W., Kirubarajan, T., and Bar-Shalom, Y. (2004). Track Segment Association, Fine-Step IMM and Initialization with Doppler for Improved Track Performance. Aerospace and Electronic Systems, IEEE Transactions on, 40(1), 293-309.

Zhang, X., Willett, P., and Bar-Shalom, Y. (2005). Dynamic Cramer-Rao Bound for Target Tracking in Clutter. Aerospace and Electronic Systems, IEEE Transactions on, 41(4), 1154-1167.

Zhou, H., Wen, B., Ma, Z., and Wu, S. (2006). Range/Doppler Ambiguity Elimination in High-Frequency Chirp Radars. Radar, Sonar and Navigation, IEE Proceedings, 153(6), 467-472. 